\title{
Thick points of high-dimensional Gaussian free fields
}

\author{
Linan Chen $^{1}$ \\ Department of Mathematics and Statistics, McGill University, 805 Sherbrooke Street West, Montréal, QC, H3A OB9, Canada. \\ E-mail: Inchen@math.mcgill.ca
}

Received 31 March 2016; revised 31 December 2016; accepted 15 May 2017

\begin{abstract}
This work aims to extend the existing results on thick points of logarithmic-correlated Gaussian Free Fields to Gaussian random fields that are more singular. To be specific, we adopt a sphere averaging regularization to study polynomial-correlated Gaussian Free Fields in higher-than-two dimensions. Under this setting, we introduce the definition of thick points which, heuristically speaking, are points where the value of the Gaussian Free Field is unusually large. We then establish a result on the Hausdorff dimension of the sets containing thick points.
\end{abstract}

Résumé. Cet article a pour but d'étendre certains résultats existants sur les points épais de champs libres gaussiens a corrélation logarithmique, à des champs aléatoires gaussiens qui sont plus singuliers. Plus précisément, nous utilisons une moyenne sphérique pour étudier les champs libres gaussiens a corrélation polynomiale en dimension supérieure à 2 . Dans ce contexte nous introduisons une définition des points épais qui, de manière heuristique, sont les points pour lesquels la valeur du champ libre gaussien est inhabituellement grande. Nous établissons un résultat sur la dimension de Hausdorff des ensembles contenant ces points épais.

MSC: 60G60; 60G15

Keywords: Gaussian free field; Polynomial singularity; Thick point; Hausdorff dimension

\section{Introduction}

Many recent developments in statistical physics and probability theory have seen Gaussian Free Field (GFF) as an indispensable tool. Heuristically speaking, GFFs are analogues of Brownian motion with multidimensional time parameters. Just as Brownian motion is thought of as a natural interpretation of "random curve", GFFs are considered as promising candidates for modeling "random surface" or "random manifold", which ultimately lead to the study of random geometry. Motivated by their importance, GFFs have been widely studied both in discrete and continuum settings, and certain geometric properties of GFFs have been revealed. For example, the distribution of extrema and near-extrema of two-dimensional log-correlated discrete GFFs are studied by Ding et al. [4,10,11]. However, for continuum GFFs, the notion of "extrema" is not applicable, because even in the two-dimensional case a generic element of the GFF is only a tempered distribution which is not defined pointwise. In fact, it is the singularity of GFFs that poses most challenges in obtaining analytic results on the geometry of GFFs. To overcome the challenges, one needs to apply a procedure, known as "regularization" in physics literature, to approximate pointwise values of GFFs in continuum settings. Various regularization procedures have been considered in the study of problems related to the geometry of log-correlated GFFs.

One such example is the theory of Gaussian multiplicative chaos (GMC) introduced by Kahane in his seminal work [18]. The GMC theory enables one to define in any dimensions random Borel measures which formally take

\footnotetext{
${ }^{1}$ Supported in part by NSERC Discovery Grant G241023.
} 
the form " $e^{h(x)} d x$ ", where $h$ is a generic element of a log-correlated Gaussian random field from a large class of such random fields, and $d x$ is the Lebesgue measure. Such measures, known as the Liouville quantum gravity (LQG) measures, are important objects in quantum field theory. Kahane's work has led to the multi-fractal analysis of the LQG measures by showing that these measures are supported on Borel sets where "unusually" large values are achieved by the regularized $h$. Over the past decade, further results, such as the exact Hausdorff dimension of the support of a LQG measure, have been established during extensive study of the LQG measures under the framework of GMC (see, e.g., [2,3,14,20-22]). Besides, using the tool of GMC, the extreme values of the regularized $h$ are treated in [19].

Apart from the GMC approach, another regularization procedure often used in the study of a continuum GFF is to average the generic field element $h$ over some sufficiently "nice" Borel sets. This is also the regularization that will be adopted in this article. Although being a tempered distribution, $h$ can still be integrated over sufficiently regular submanifolds. Since convolution or integration is the natural way to "tame" the singularity of a tempered distribution, such an averaging procedure becomes a natural choice when it comes to the study of the "landscape" of $h$. For example, for $h$ being a log-correlated GFF in 2D, by considering the averages of $h$ over circles, Duplantier and Sheffield [15] also provided a rigorous construction of the LQG measures in 2D and derived the same property for the supports of the LQG measures as mentioned above, i.e., such measures are supported on sets where the averaged $h$ achieves "unusually" large values. Meanwhile, using the same regularization, i.e., circular averages of $h$, $\mathrm{Hu}$, Miller and Peres [17] studied specifically the points where the averaged $h$ is "unusually" large, and introduced the terminology of "thick points"2 for such points.

More specifically, let $h$ be a generic element of the GFF associated with the operator $\Delta$ on a bounded domain $D \subseteq \mathbb{R}^{2}$ with the Dirichlet boundary condition. Governed by the properties of the Green's function of $\Delta$ in $2 \mathrm{D}$, such a GFF is logarithmically correlated, and it is possible to interpret, in the sense of random variable, the circular average of $h$ :

$$
\bar{h}_{t}(z):=\frac{1}{2 \pi t} \int_{\partial B(z, t)} h(x) \sigma(d x),
$$

where $z \in D, \partial B(z, t)$ is the circle centered at $z$ with radius $t$ and $\sigma(d x)$ is the length measure along the circle. To get an approximation of " $h(z)$ ", it is to our interest to study $\bar{h}_{t}(z)$ as $t \searrow 0$. For every $a \geq 0$, the set of $a$-thick points of $h$ are defined in [17] as

$$
T_{h}^{a}:=\left\{z \in D: \lim _{t \searrow 0} \frac{\bar{h}_{t}(z)}{(-\ln t)}=\sqrt{\frac{a}{\pi}}\right\} .
$$

With $z$ fixed, the circular average process $\left\{\bar{h}_{t}(z): z \in(0,1]\right\}$ has the same distribution as a Brownian motion $\left\{B_{\tau}(z)\right.$ : $\tau \geq 0\}$ up to a deterministic time change $\tau=(-\ln t) / \sqrt{2 \pi}$, and as $t \searrow 0, \bar{h}_{t}(z)$ behaves just like $B_{\tau}(z)$ as $\tau \nearrow \infty$. Then, for any given $z \in D$, written in terms of $\left\{B_{\tau}(z): \tau \geq 0\right\}$, the limit involved in (1.1) is equivalent to

$$
\lim _{\tau \rightarrow \infty} \frac{B_{\tau}(z)}{\tau}=\sqrt{2 a}
$$

which occurs with probability zero for any $a>0$. Therefore, $a$-thick points, so long as $a>0$, are locations where the field value is "unusually" large. The authors of [17] prove that for every $a \in[0,2], \operatorname{dim}_{\mathcal{H}}\left(T_{h}^{a}\right)=2-a$ a.s., where " $\operatorname{dim}_{\mathcal{H}}$ " denotes the Hausdorff dimension.

Besides being the support of the LQG measures, thick points themselves characterize a fundamental aspect of the "landscape" of GFFs, that is, where the "high peaks" occur. Hence, thick points are of importance to understanding the geometry of GFFs. By adapting the circle averaging regularization introduced above, certain results from [15] and [17] on log-correlated GFFs have been extended from 2D to higher dimensions. For example, Chen and Jakobson [5] treated the LQG measure associated with a log-correlated GFF in higher even dimensions via a specific functional of spherical averages of the field; based on similar regularization, Cipriani and Hazra [7,8] investigated the thick points of higher dimensional log-correlated GFFs. It is shown that for log-correlated GFFs in any dimensions, one

\footnotetext{
${ }^{2}$ The term "thick point" is borrowed from the literature of stochastic analysis. There it refers to the extremes of the occupation measure of a stochastic process (see, e.g., [9]).
} 
can similarly define thick point sets as in (1.1) and a result on Hausdorff dimensions of such sets is in order. However, to the best of the author's knowledge, there had been no comparable study of thick points for GFFs that are more singular, e.g., polynomial-correlated GFFs. In fact, to date little is known about the geometry of such GFFs. ${ }^{3}$ Inspired by the approach presented in [17], this article lays out the first step of an attempt to explore geometric problems associated with polynomial-correlated GFFs in any dimensions.

The main focus of this article is to extend the techniques and the results on thick points of log-correlated GFFs to polynomial-correlated GFFs on $\mathbb{R}^{v}$ for any $v>2$. Intuitively speaking, compared with the log-correlated counterparts, GFFs that are polynomially correlated consist of generic elements that are more singular so the "landscape" of such a field is "rougher", and the higher the dimension $v$ is, the worse it becomes. To make these remarks rigorous and to bring generality to our approach, we adopt the theory of the Abstract Wiener Space [16] to interpret general Gaussian random fields, including GFFs with any degree of polynomial singularity in any dimensions. Let $\theta$ be a generic element of such a field. It is always possible, by averaging $\theta$ over codimension- 1 spheres centered at $x \in \mathbb{R}^{v}$, to obtain a proper approximation $\bar{\theta}_{t}(x)$ which approaches " $\theta(x)$ " as $t \searrow 0$. We give a careful analysis of the two parameter Gaussian family

$$
\left\{\bar{\theta}_{t}(x): x \in \mathbb{R}^{v}, t \in(0,1]\right\}
$$

and use the concentric spherical averages (with $x$ fixed) to define thick points. It turns out that, instead of the most straightforward analogue of (1.1), the suitable definition for thick points of the degree- $(v-2)$-polynomial-correlated GFF is that, for $\gamma \geq 0, x$ is a $\gamma$-thick point of $\theta$ if and only if

$$
\limsup _{t \searrow 0} \frac{\bar{\theta}_{t}(x)}{\sqrt{-G(t) \ln t}} \geq \sqrt{2 v \gamma},
$$

where $G(t):=\mathbb{E}\left[\left(\bar{\theta}_{t}(x)\right)^{2}\right]$. In a similar spirit as (1.1), if $\gamma>0$, then a $\gamma$-thick point is a location where $\theta$ is unusually large. By adapting the approach presented in [17], we establish the result (Theorem 9) that if $T_{\theta}^{\gamma}$ is the set consisting of all the $\gamma$-thick points of $\theta$ in the unit cube in $\mathbb{R}^{\nu}$, then

$$
\operatorname{dim}_{\mathcal{H}}\left(T_{\theta}^{\gamma}\right)=v(1-\gamma) \text { a.s. }
$$

Moreover, we investigate the relation between (1.1) and (1.2), and show that (Theorem 13) due to the higher-order singularity of the polynomial-correlated GFFs, with probability one, the "perfect" $\gamma$-thick point, i.e., $x$ such that

$$
\lim _{t \searrow 0} \frac{\bar{\theta}_{t}(x)}{\sqrt{-G(t) \ln t}}=\sqrt{2 \nu \gamma},
$$

does not exist, which explains why (1.2) is more suitable a choice than (1.1) as the definition of thick point for GFFs that are polynomially correlated. On the other hand, if we relax the condition in (1.3) to

$$
\lim _{n \rightarrow \infty} \frac{\bar{\theta}_{r_{n}}(x)}{\sqrt{-G\left(r_{n}\right) \ln r_{n}}}=\sqrt{2 \nu \gamma},
$$

where $\left\{r_{n}: n \geq 0\right\}$ is any sequence that decays to zero sufficiently fast, then we find out (Theorem 16) that, if $S T_{\theta}^{\gamma}$ is the set consisting of all the points $x$ in the unit cube in $\mathbb{R}^{v}$ that satisfies (1.4), then

$$
\operatorname{dim}_{\mathcal{H}}\left(S T_{\theta}^{\gamma}\right)=v(1-\gamma) \text { a.s. }
$$

Some lemmas we obtained during the process are of independent interest.

In Section 2 we briefly introduce the theory of the Abstract Wiener Space as the foundation for the study of GFFs. In Section 3 we give a detailed study of the Gaussian family consisting of spherical averages of the GFFs. These are

\footnotetext{
${ }^{3}$ In the discrete setting, it is possible to realize on $\mathbb{Z}^{v}(v \geq 3)$ a polynomial-correlated discrete GFF via a random-coefficient series of eigenfunctions of Laplacian on $\mathbb{Z}^{v}$. In that realm related problems such as percolation models associated with level sets of discrete GFFs have been studied (e.g., [12] and the references therein).
} 
the main tools that will be exploited in later parts of this article. Our main results are stated in Section 4 and at the beginning of Section 5 . In particular, the result on $\operatorname{dim}_{\mathcal{H}}\left(T_{\theta}^{\gamma}\right)$ are proved by establishing the upper bound and the lower bound separately. The upper bound is proved in Section 4.1, and the lower bound is established in multiple steps in Section 5.

\section{Abstract Wiener space and Gaussian free fields}

The theory of Abstract Wiener Space (AWS), first introduced by Gross [16], provides an analytical foundation for the construction and the study of Gaussian measures in infinite dimensions. To be specific, given a real separable Banach space $E$, a non-degenerate centered Gaussian measure $\mathcal{W}$ on $E$ is a Borel probability measure such that for every $x^{*} \in E^{*} \backslash\{0\}$, the functional $x \in E \mapsto\left\langle x, x^{*}\right\rangle \in \mathbb{R}$ has non-degenerate centered Gaussian distribution under $\mathcal{W}$, where $E^{*}$ is the space of bounded linear functionals on $E$, and $\left\langle\cdot, x^{*}\right\rangle$ is the action of $x^{*} \in E^{*}$ on $E$. Further assume that $H$ is a real separable Hilbert space which is continuously embedded in $E$ as a dense subspace. Then $E^{*}$ can be continuously and densely embedded into $H$, and for every $x^{*} \in E^{*}$ there exists a unique $h_{x^{*}} \in H$ such that $\left\langle h, x^{*}\right\rangle=\left(h, h_{x^{*}}\right)_{H}$ for all $h \in H$. Under this setting if the Gaussian measure $\mathcal{W}$ on $E$ has the following characteristic function:

$$
\mathbb{E}^{\mathcal{W}}\left[\exp \left(i\left\langle\cdot, x^{*}\right\rangle\right)\right]=\exp \left(-\frac{\left\|h_{x^{*}}\right\|_{H}^{2}}{2}\right) \text { for every } x^{*} \in E^{*},
$$

then the triple $(H, E, \mathcal{W})$ is called an Abstract Wiener Space. Moreover, since $\left\{h_{x^{*}}: x^{*} \in E^{*}\right\}$ is dense in $H$, the mapping

$$
\mathcal{I}: h_{x^{*}} \in H \mapsto \mathcal{I}\left(h_{x^{*}}\right):=\left\langle\cdot, x^{*}\right\rangle \in L^{2}(\mathcal{W})
$$

can be uniquely extended to a linear isometry between $H$ and $L^{2}(\mathcal{W})$. The extended isometry, also denoted by $\mathcal{I}$, is the Paley-Wiener map and its images $\{\mathcal{I}(h): h \in H\}$, known as the Paley-Wiener integrals, form a centered Gaussian family whose covariance is given by

$$
\mathbb{E}^{\mathcal{W}}[\mathcal{I}(h) \mathcal{I}(g)]=(h, g)_{H} \quad \text { for all } h, g \in H .
$$

Therefore, if $\left\{h_{n}: n \geq 1\right\}$ is an orthonormal basis of $H$, then $\left\{\mathcal{I}\left(h_{n}\right): n \geq 1\right\}$ is family of i.i.d. standard Gaussian random variables. In fact,

$$
\text { for } \mathcal{W} \text {-a.e. } x \in E, \quad x=\sum_{n \geq 1} \mathcal{I}\left(h_{n}\right)(x) h_{n} .
$$

Although $\mathcal{W}$ is a measure on $E$, it is the inner product of $H$ that determines the covariance structure of $\mathcal{W} . H$ is referred to as the Cameron-Martin space of $(H, E, \mathcal{W})$. The theory of AWS says that given any separable Hilbert space $H$, one can always find $E$ and $\mathcal{W}$ such that the triple $(H, E, \mathcal{W})$ forms an AWS. On the other hand, given a separable Banach space $E$, any non-degenerate centered Gaussian measure $\mathcal{W}$ on $E$ must exist in the form of an AWS. That is to say that, AWS is the "natural" format in which any infinite dimensional Gaussian measure exists. For further discussions on the construction and the properties of AWS, we refer to [6,16,24] and Chapter 8 of [25].

We now apply the general theory of AWS to study Gaussian measures on function or generalized function spaces. To be specific, given $v \in \mathbb{N}$ and $p \in \mathbb{R}$, consider the Sobolev space $H^{p}:=H^{p}\left(\mathbb{R}^{v}\right)$, which is the closure of $C_{c}^{\infty}\left(\mathbb{R}^{v}\right)$, the space of compactly supported smooth functions on $\mathbb{R}^{v}$, under the inner product given by, for every $\phi, \psi \in C_{c}^{\infty}\left(\mathbb{R}^{\nu}\right)$,

$$
\begin{aligned}
(\phi, \psi)_{H^{p}} & :=\left((I-\Delta)^{p} \phi, \psi\right)_{L^{2}\left(\mathbb{R}^{v}\right)} \\
& =\frac{1}{(2 \pi)^{v}} \int_{\mathbb{R}^{v}}\left(1+|\xi|^{2}\right)^{p} \hat{\phi}(\xi) \overline{\hat{\psi}(\xi)} d \xi
\end{aligned}
$$

where $\hat{\bullet}$ denotes the Fourier transform. $\left(H^{P},(\cdot, \cdot)_{H^{p}}\right)$ is a separable Hilbert space, and it will be taken as the CameronMartin space for the discussions in this article. As mentioned earlier, there exists a separable Banach space $\Theta^{p}:=$ 
$\Theta^{p}\left(\mathbb{R}^{v}\right)$ and a Gaussian measure $\mathcal{W}^{p}:=\mathcal{W}^{p}\left(\mathbb{R}^{v}\right)$ on $\Theta^{p}$ such that the triple $\left(H^{p}, \Theta^{p}, \mathcal{W}^{p}\right)$ forms an AWS, to which we refer as the dim-v order-p Gaussian Free Field (GFF). ${ }^{4}$ It is clear that the covariance of such a field is determined by the Green's function of $(I-\Delta)^{p}$ on $\mathbb{R}^{v}$.

To give explicit formulations for the GFFs introduced in the framework above, we review the result in [25] (Section 8.5) that, when $p=\frac{v+1}{2}, \Theta^{\frac{v+1}{2}}$ can be taken as

$$
\Theta^{\frac{v+1}{2}}:=\left\{\theta \in C\left(\mathbb{R}^{\nu}\right): \lim _{|x| \rightarrow \infty} \frac{|\theta(x)|}{\log (e+|x|)}=0\right\},
$$

equipped with the norm

$$
\|\theta\|_{\Theta^{\frac{v+1}{2}}}:=\sup _{x \in \mathbb{R}^{\nu}} \frac{|\theta(x)|}{\log (e+|x|)} .
$$

In other words, the dim- $v$ order- $\frac{v+1}{2}$ GFF consists of continuous functions on $\mathbb{R}^{v}$. More generally, for $p \in \mathbb{R}, H^{p}$ is the isometric image of $H^{\frac{v+1}{2}}$ under the Bessel-type operator $(I-\Delta)^{\frac{v+1-2 p}{4}}$. Therefore, we can take $\Theta^{p}$ to be image of $\Theta^{\frac{v+1}{2}}$ under $(I-\Delta)^{\frac{v+1-2 p}{4}}$ and the corresponding Gaussian measure is

$$
\mathcal{W}^{p}=\left((I-\Delta)^{-\frac{v+1-2 p}{4}}\right)_{\star} \mathcal{W}^{\frac{v+1}{2}}
$$

In addition, if we identify $H^{-p}$ as the dual space of $H^{p}$, then $\left(\Theta^{p}\right)^{*} \subseteq H^{-p}$ and for every $\lambda \in\left(\Theta^{p}\right)^{*}$, it is easy to see that

$$
\lambda \mapsto h_{\lambda}:=(I-\Delta)^{-p} \lambda
$$

gives the unique element $h_{\lambda} \in H^{p}$ such that the action of $\lambda \in\left(\Theta^{p}\right)^{*}$, when restricted on $H^{p}$, coincides with $\left(\cdot, h_{\lambda}\right)_{H^{p}}$. Moreover, the map (2.2) can also be viewed as an isometry between $H^{-p}$ and $H^{p}$. For $\lambda \in H^{-p}$, we still use " $h_{\lambda}$ " to denote the image of $\lambda$ under (2.2). Then the Paley-Wiener integrals $\left\{\mathcal{I}\left(h_{\lambda}\right): \lambda \in H^{-p}\right\}$ form a centered Gaussian family with the covariance

$$
\mathbb{E}^{\mathcal{W} \mathcal{W}^{p}}\left[\mathcal{I}\left(h_{\lambda}\right) \mathcal{I}\left(h_{\eta}\right)\right]=\left(h_{\lambda}, h_{\eta}\right)_{H^{p}}=(\lambda, \eta)_{H^{-p}} \quad \text { for every } \lambda, \eta \in H^{-p} .
$$

It is clear from the discussions above that with the dimension $v$ fixed, the larger the order $p$ is, the more regular the elements of the GFF are; on the other hand, if $p$ is fixed, then the higher the dimension $v$ is, the more singular the GFF becomes. In most of the cases that are of interest to us, generic elements of GFFs are only tempered distributions. For example, this is the case with GFFs that are logarithmically correlated. Interpreted under the framework introduced above, log-correlated GFFs are dim- $v$ order-( $v / 2)$ GFFs, i.e., with $p=v / 2$, since the Green's function of $(I-\Delta)^{v / 2}$ on $\mathbb{R}^{v}$ has logarithmic singularity along the diagonal. On the other hand, if $2 p \in \mathbb{N}$ and $2 p<v$, the Green's function have polynomial singularity with degree $v-2 p$ and hence the corresponding GFFs are polynomially correlated. In this article, we focus on studying certain geometric properties of polynomial-correlated GFFs with $^{5} p \in \mathbb{N}$ and $p<v / 2$.

We finish this section by remarking that, instead of using the Bessel-type operator $(I-\Delta)^{p}$ to construct GFFs on $\mathbb{R}^{v}$, one can also use the operator $\Delta^{p}$, equipped with proper boundary conditions, to construct GFFs on bounded domains on $\mathbb{R}^{v}$ (e.g., [15,17] and [23]). The field elements obtained in either way possess similar local properties. However, $(I-\Delta)^{p}$ rather than $\Delta^{p}$ is a better choice for this project for the technical reason that $(I-\Delta)^{p}$ allows the GFF to be defined on the entire space, and hence we don't have to specify a boundary condition, which is an advantage at least when $p>1$.

\footnotetext{
${ }^{4}$ In physics literature, the term "GFF" only refers to the case when $p=1$. Here we slightly extend the use of this term and continue to use GFF.

${ }^{5}$ The GFFs with $p$ being half integers (and hence the operator is non-local) are considered by O. Nadeau-Chamard and the author in a separate paper which is currently in preparation.
} 


\section{Spherical averages of Gaussian free fields}

For the rest of this article, we assume that $v, p \in \mathbb{N}, v>2$ and $1 \leq p<v / 2$, and $\theta$ is a generic element of the dim$v$ order- $p$ GFF, i.e., $\theta \in \Theta^{p}$ is sampled under $\mathcal{W}^{p}$. Although " $\theta(x)$ " is not defined for every $x \in \mathbb{R}^{v}$, we can use the "average" of $\theta$ over a sphere centered at $x$ to approximate " $\theta(x)$ ", as the radius of the sphere tends to zero. To make this precise, we need to introduce some notation. Let $B(x, t)$ and $\partial B(x, t)$ be the open ball and, respectively, the sphere centered at $x \in \mathbb{R}^{v}$ with radius (under the Euclidean metric) $t>0, \sigma_{x, t}$ the surface measure on $\partial B(x, t)$, $\alpha_{v}(t):=\alpha_{\nu} t^{\nu-1}$ the surface area of $\partial B(x, t)$ with $\alpha_{v}:=2\left(\pi^{\nu / 2}\right) / \Gamma(\nu / 2)$, and $\sigma_{x, t}^{\text {ave }}:=\sigma_{x, t} / \alpha_{\nu}(t)$ the spherical average measure over $\partial B(x, t)$. We first state the following simple facts about $\sigma_{x, t}^{\text {ave }}$. It is straightforward to derive these results, so we will omit the proofs.

Lemma 1. For every $x \in \mathbb{R}^{v}$ and $t>0, \sigma_{x, t}^{\text {ave }} \in H^{-1}\left(\mathbb{R}^{v}\right)$ and its Fourier transform is given by

$$
\forall \xi \in \mathbb{R}^{v}, \quad \widehat{\sigma}_{x, t}^{\mathrm{ave}}(\xi)=\frac{(2 \pi)^{\frac{v}{2}}}{\alpha_{\nu}} e^{i(x, \xi)_{\mathbb{R}^{\nu}}} \cdot(t|\xi|)^{\frac{2-v}{2}} J_{\frac{v-2}{2}}(t|\xi|)
$$

where $J_{\frac{v-2}{2}}$ is the standard Bessel function of the first kind with index $\frac{v-2}{2}$.

The first assertion of the lemma implies that $\sigma_{x, t}^{\text {ave }} \in H^{-p}\left(\mathbb{R}^{v}\right)$ for every $p \geq 1$. In particular, this fact shows that, no matter what the dimension is and how singular the GFF is, a codimension- 1 sphere is always sufficiently "nice" that it is possible to average the GFF over such a sphere. As a consequence, $\mathcal{I}\left(h_{\sigma_{x, t}^{\text {ave }}}\right)$, viewed as the spherical average of the GFF, is well defined for every $x \in \mathbb{R}^{v}$ and $t>0$ as a Gaussian random variable, and as $t \searrow 0$, from the point of the view of tempered distributions, $\mathcal{I}\left(h_{\sigma_{x, t}}^{\text {ave }}\right)(\theta)$ approximates " $\theta(x)$ ". With the help of (3.1), we can compute, by Parseval's identity, the covariance of the Gaussian family consisting of all the spherical averages and express the covariance as follows.

Lemma 2. $\left\{\mathcal{I}\left(h_{\sigma_{x, t}^{\text {ave }}}\right): x \in \mathbb{R}^{v}, t>0\right\}$ is a two-parameter centered Gaussian family under $\mathcal{W}^{p}$, and the covariance is given by

$$
\begin{aligned}
\mathbb{E}^{\mathcal{W}} & {\left[\mathcal{I}\left(h_{\sigma_{x, t}^{\text {ave }}}\right) \mathcal{I}\left(h_{\sigma_{y, s}^{\text {ave }}}\right)\right] } \\
= & \frac{(2 \pi)^{v / 2}}{\alpha_{v}^{2}(t s|x-y|)^{\frac{v-2}{2}}} \int_{0}^{\infty} \frac{\tau^{2-\frac{v}{2}} J_{\frac{v-2}{2}}(t \tau) J_{\frac{\nu-2}{2}}(s \tau) J_{\frac{\nu-2}{2}}(|x-y| \tau)}{\left(1+\tau^{2}\right)^{p}} d \tau .
\end{aligned}
$$

In particular, when $x=y$, i.e., in the case of concentric spherical averages,

$$
\mathbb{E}^{\mathcal{W} \mathcal{W}^{p}}\left[\mathcal{I}\left(h_{\sigma_{x, t}^{\text {ave }}}\right) \mathcal{I}\left(h_{\sigma_{x, s}^{\text {ave }}}\right)\right]=\frac{1}{\alpha_{\nu}(t s)^{\frac{\nu-2}{2}}} \int_{0}^{\infty} \frac{\tau J_{\frac{v-2}{2}}(t \tau) J_{\frac{v-2}{2}}(s \tau)}{\left(1+\tau^{2}\right)^{p}} d \tau .
$$

Again, these results follow easily from integral representations of Bessel functions ([27], Section 3.3) combined with straightforward computations. Proofs are omitted.

To study the distribution of the family of spherical averages, and to use them effectively to approximate "pointwise values" of the GFF, it is useful to obtain as explicit an expression for the covariance as possible. To this end, we will first assume $p=1$ and treat the concentric spherical averages (Section 3.1) and the non-concentric ones (Section 3.2) separately. During this process, we find for each $x \in \mathbb{R}^{v}$ a set of "renormalized spherical averages" which still approximates " $\theta(x)$ " but whose covariance has technically desirable properties. In Section 3.3 we briefly explain the strategy for treating the spherical averages when $p>1$.

\subsection{When $p=1$. Concentric spherical averages}

For the rest of this article, when $p=1$, we simply write $\left(H^{p}, \Theta^{p}, \mathcal{W}^{p}\right)$ as $(H, \Theta, \mathcal{W})$. It is clear from (3.3) that the distribution of the concentric spherical averages $\left\{\mathcal{I}\left(h_{\sigma_{x, t} \text { ave }}\right): t>0\right\}$ at any given $x \in \mathbb{R}^{v}$ is independent of $x$. In fact, the distribution of the GFF is translation invariant. First we state a closed formula for the integral in (3.3). 
Lemma 3. Fix any $x \in \mathbb{R}^{v}$. For every $t, s>0$,

$$
\mathbb{E}^{\mathcal{W}}\left[\mathcal{I}\left(h_{\sigma_{x, t}^{\mathrm{ave}}}\right) \mathcal{I}\left(h_{\sigma_{x, s}^{\mathrm{ave}}}\right)\right]=\frac{1}{\alpha_{\nu}(t s)^{\frac{\nu-2}{2}}} I_{\frac{\nu-2}{2}}(t \wedge s) K_{\frac{v-2}{2}}(t \vee s)
$$

where $I_{\frac{v-2}{2}}$ and $K_{\frac{v-2}{2}}$ are the modified Bessel functions (with pure imaginary argument) with the index $\frac{v-2}{2}$.

One can use a formula in [27] (Section 13.53) to derive (3.4) directly. An alternative proof was provided in the Appendix of [5]. ${ }^{6}$ So we will omit the proof of Lemma 3 and refer to [27] and [5] for details.

By (3.4), $\left\{\mathcal{I}\left(h_{\sigma_{x, t}^{\text {ave }}}\right): t>0\right\}$ is a backward ${ }^{7}$ Markov Gaussian process. In fact, (3.4) leads to a renormalization of the spherical averages. Namely, if

$$
\bar{\sigma}_{x, t}:=\frac{(t / 2)^{\frac{v-2}{2}}}{\Gamma(\nu / 2) \cdot I_{\frac{v-2}{2}}(t)} \cdot \sigma_{x, t}^{\text {ave }},
$$

then $\bar{\sigma}_{x, t} \in H^{-1}$. Let $\bar{\theta}_{t}(x)$ be the corresponding Paley-Wiener integral $\mathcal{I}\left(h_{\bar{\sigma}_{x, t}}\right)(\theta)$. It is easy to verify that

$$
\lim _{t \rightarrow 0} \frac{(t / 2)^{\frac{v-2}{2}}}{\Gamma(\nu / 2) \cdot I_{\frac{v-2}{2}}(t)}=1,
$$

so as $t \searrow 0, \bar{\theta}_{t}(x)$ still is a legitimate approximation of " $\theta(x)$ ". It follows from (3.4) that the covariance of the Gaussian process $\left\{\bar{\theta}_{t}(x): t>0\right\}$ is given by, for $0<s \leq t$,

$$
\mathbb{E}^{\mathcal{W}}\left[\bar{\theta}_{t}(x) \bar{\theta}_{s}(x)\right]=\frac{\alpha_{v}}{(2 \pi)^{v}} \cdot \frac{K_{\frac{v-2}{2}}(t)}{I_{\frac{v-2}{2}}(t)}=: G(t) .
$$

The function $G$ defined above is positive and decreasing on $(0, \infty)$, and when $t$ is sufficiently small, $G(t)=\mathcal{O}\left(t^{2-v}\right)$, which reflects the fact that the dim- $v$ order-1 GFF is polynomially correlated with degree $v-2$.

Remark 4. Since we are only concerned about $\bar{\theta}_{t}(x)$ when $t$ is small, without loss of generality, we assume that $t \in(0,1]$. As a consequence of $(3.5),\left\{\bar{\theta}_{t}(x): t \in(0,1]\right\}$ is a Gaussian process with independent increment (in the direction of $t$ decreasing), which, up to a time change, has the same distribution as a Brownian motion. To be specific, if we define a "clock" by

$$
\tau:=G(t)-G(1) \text { for } t \in(0,1],
$$

then

$$
\left\{B_{\tau}:=\bar{\theta}_{G^{-1}(\tau+G(1))}(x)-\bar{\theta}_{1}(x): \tau \geq 0\right\}
$$

has the same distribution as a standard Brownian motion.

Based on the preceding observations, results about the Brownian motion can be transported directly to $\left\{\bar{\theta}_{t}(x): t \in\right.$ $(0,1]\}$, and the behavior of $\bar{\theta}_{t}(x)$ when $t$ is small resembles that of the Brownian motion $B_{\tau}$ when $\tau$ is large. For example, by the law of the iterated logarithm,

$$
\limsup _{t \searrow 0} \frac{\left|\bar{\theta}_{t}(x)\right|}{\sqrt{2 G(t) \cdot \ln \ln G(t)}}=1 \quad \text { a.s. }
$$

\footnotetext{
${ }^{6}$ The formula (3.4), as well as (3.7) and (3.8) below, are proven in the Appendix in [5] for the case when $v=4$, but the proof is identical in any dimensions.

${ }^{7}$ A "backward" Markov process is a process which exhibits Markov property as the parameter " $t$ " decreases.
} 


\subsection{When $p=1$. Non-concentric spherical averages}

We now move on to the family of non-concentric spherical averages. Again, instead of the regular spherical averages, we adopt the renormalized spherical averages introduced in Section 3.1. Consider the two-parameter Gaussian family

$$
\left\{\bar{\theta}_{t}(x): x \in \mathbb{R}^{v}, t \in(0,1]\right\},
$$

and denote by $\operatorname{Cov}(x, t ; y, s)$ the covariance of $\bar{\theta}_{t}(x)$ and $\bar{\theta}_{s}(y)$. One can compute $\operatorname{Cov}(x, t ; y, s)$ using (3.2) and the renormalization. In fact, under certain circumstances, it is possible to obtain explicit formulas for $\operatorname{Cov}(x, t ; y, s)$.

Lemma 5. Let $x, y \in \mathbb{R}^{v}$ and $t, s \in(0,1]$.

(i) If $|x-y| \geq t+s$, i.e., if $B(x, t) \cap B(y, s)=\varnothing$,

$$
\operatorname{Cov}(x, t ; y, s)=(2 \pi)^{-v / 2} \cdot \frac{K_{\frac{v-2}{2}}(|x-y|)}{|x-y|^{\frac{v-2}{2}}}=: C_{\text {disj }}(|x-y|) .
$$

In particular, $C_{\mathrm{disj}}(|x-y|)=\mathcal{O}\left(|x-y|^{2-v}\right)$ when $|x-y|$ is small.

(ii) If $t \geq|x-y|+s$, i.e., if $B(x, t) \supset B(y, s)$,

$$
\operatorname{Cov}(x, t ; y, s)=(2 \pi)^{-v / 2} \cdot \frac{I_{\frac{v-2}{2}}(|x-y|)}{|x-y|^{\frac{v-2}{2}}} \cdot \frac{K_{\frac{v-2}{2}}(t)}{I_{\frac{v-2}{2}}(t)}=: C_{\text {incl }}(t,|x-y|) .
$$

In particular, $C_{\text {incl }}(t,|x-y|)=\mathcal{O}\left(t^{2-v}\right)$ when $t$ is small.

Again, by combining (3.2) with a formula in [27] (Section 13.53, pp. 429-430), one can easily verify these results. An alternative derivation was also provided in the Appendix of [5]. We omit the proofs and refer to [27] and [5] for details. We remark that (3.7) and (3.8) demonstrate the advantage of this particular renormalization of the spherical averages. For the family of the renormalized spherical averages, under the hypothesis (i) or (ii) in Lemma 5, small radius (radii) does not affect the covariance, which favors convergence as radius (radii) tends to zero.

However, one still needs to treat the renormalized spherical averages in the most general case. To this end, we introduce the intrinsic metric $d$ associated with the Gaussian family $\left\{\bar{\theta}_{t}(x): x \in \mathbb{R}^{v}, t \in(0,1]\right\}$ where

$$
d(x, t ; y, s):=\left(\mathbb{E}^{\mathcal{W}}\left[\left|\bar{\theta}_{t}(x)-\bar{\theta}_{s}(y)\right|^{2}\right]\right)^{\frac{1}{2}}
$$

for $x, y \in \mathbb{R}^{v}$ and $t, s \in(0,1]$. Assuming $0<s \leq t \leq 1$, the triangle inequality implies that

$$
d(x, t ; y, s) \leq d(x, t ; y, t)+\sqrt{G(s)-G(t)},
$$

so to work with $d(x, t ; y, s)$, we need to study $d(x, t ; y, t)$, i.e., the intrinsic metric associated with the family $\left\{\bar{\theta}_{t}(x)\right.$ : $\left.x \in \mathbb{R}^{v}\right\}$ at any fixed $t \in(0,1]$.

Lemma 6. There exists a constant ${ }^{8} C_{v}>0$ such that for every $t \in(0,1]$ and every $x, y \in \mathbb{R}^{v}$,

$$
d^{2}(x, t ; y, t) \leq C_{v} \cdot t^{2-v}\left(\sqrt{\frac{|x-y|}{t}} \wedge 1\right)
$$

Proof. Based on (3.7), when $|x-y| \geq 2 t$,

$$
d^{2}(x, t ; y, t)=2 G(t)-2 C_{\mathrm{disj}}(|x-y|),
$$

\footnotetext{
${ }^{8}$ Throughout the article, $C_{v}$ denotes a constant that only depends on the dimension, and $C_{v}$ 's value may vary from line to line.
} 
which immediately implies (3.10). More generally, using (3.2) and (3.3), we can rewrite $d^{2}(x, t ; y, t)$ as

$$
\begin{aligned}
d^{2}(x, t ; y, t) & =\mathbb{E}^{\mathcal{W}}\left[\left(\bar{\theta}_{t}(x)-\bar{\theta}_{t}(y)\right)^{2}\right] \\
& =\frac{2 \alpha_{v}}{(2 \pi)^{v} I_{\frac{v-2}{2}}^{2}(t)} \int_{0}^{\infty} \frac{\tau}{1+\tau^{2}} J_{\frac{v-2}{2}}^{2}(t \tau) \Psi(\tau|x-y|) d \tau,
\end{aligned}
$$

where $\Psi$ is the function given by

$$
\forall w \in(0, \infty), \quad \Psi(w):=1-\frac{(2 \pi)^{v / 2}}{\alpha_{v}} w^{\frac{2-v}{2}} J_{\frac{v-2}{2}}(w) .
$$

It follows from the properties of $J_{\frac{v-2}{2}}$ that $\Psi$ is analytic and

$$
\Psi(w)=\Gamma(v / 2) \sum_{m=1}^{\infty} \frac{(-1)^{m-1} 2^{-2 m}}{m ! \Gamma\left(\frac{v}{2}+m\right)} \cdot w^{2 m} .
$$

Clearly, there exists $C_{v}>0$ such that $|\Psi(w)| \leq C_{v} \sqrt{w}$ for all $w \in[0, \infty)$. Therefore,

$$
d^{2}(x, t ; y, t) \leq C_{v} \cdot t^{2-v} \sqrt{|x-y|} \int_{0}^{\infty} \frac{\tau^{3 / 2}}{1+\tau^{2}} J_{\frac{v-2}{2}}^{2}(t \tau) d \tau,
$$

and the integral on the right, after a change of variable $u=t \tau$, becomes

$$
t^{-1 / 2} \int_{0}^{\infty} \frac{u^{3 / 2}}{t^{2}+u^{2}} J_{\frac{v-2}{2}}^{2}(u) d u \leq t^{-1 / 2} \int_{0}^{\infty} u^{-1 / 2} J_{\frac{v-2}{2}}^{2}(u) d u=C_{v} \cdot t^{-1 / 2},
$$

which leads to the desired inequality.

Based on (3.9) and (3.10), it follows from the Kolmogorov continuity theorem that there exists a continuous modification of $\left\{\bar{\theta}_{t}(x): x \in \mathbb{R}^{v}, t \in(0,1]\right\}$. From now on, we assume that $\left\{\bar{\theta}_{t}(x): x \in \mathbb{R}^{v}, t \in(0,1]\right\}$ is such a modification. In other words, we assume that for every $\theta \in \Theta,(x, t) \in \mathbb{R}^{v} \times(0,1] \mapsto \bar{\theta}_{t}(x) \in \mathbb{R}$ is continuous.

Since the distribution of the GFF is translation invariant and the notion of "thick point" only concerns local properties of the GFF, without loss of generality, we may restrict the GFF to $\overline{S(O, 1)}$ the closed cube centered at the origin with side length 2 under the Euclidean metric. ${ }^{9}$ We will apply the metric entropy method $[1,13,26]$ to study the boundedness and the continuity of the family $\left\{\bar{\theta}_{t}(x): x \in \overline{S(O, 1)}, t \in(0,1]\right\}$. To set this up, we need to introduce some more notation. For every compact subset $A \subseteq \overline{S(O, 1)} \times(0,1]$, let $\operatorname{diam}_{d}(A)$ be the diameter of $A$ under the metric $d$. $A$ is also compact under $d$, so $A$ can be finitely covered under $d$. For $\epsilon>0$ and $\mathbf{x} \in \overline{S(O, 1)} \times(0,1]$, let $B_{d}(\mathbf{x}, \epsilon)$ be the open ball centered at $\mathbf{x}$ with radius $\epsilon$ under $d$, and $N(\epsilon, A)$ the smallest number of such balls $B_{d}(\mathbf{x}, \epsilon)$ required to cover $A$. Then $N$ is the metric entropy function with respect to $d$. Applying the standard entropy methods, we get the following results.

Lemma 7. There exists a constant $C_{v}>0$ such that for every $t, s \in(0,1]$ and every $x \in \overline{S(O, 1)}$,

$$
\mathbb{E}^{\mathcal{W}}\left[\sup _{y \in \overline{S(x, s)}}\left|\bar{\theta}_{t}(y)\right|\right] \leq C_{v} \cdot t^{1-v / 2}\left(\frac{s^{1 / 4}}{t^{1 / 4}} \wedge \sqrt{\ln \left(\frac{s}{t}\right)}\right) .
$$

Proof. By (3.10), there exists $C_{v}>0$ such that for every $y, y^{\prime} \in \overline{S(x, s)}$,

$$
d\left(y, t ; y^{\prime}, t\right) \leq C_{v} \cdot t^{1-v / 2}\left(\frac{\left|y-y^{\prime}\right|^{1 / 4}}{t^{1 / 4}} \wedge 1\right) .
$$

${ }^{9}$ Similarly, for $x \in \overline{S(O, 1)}$ and $s>0, \overline{S(x, s)}$ is the Euclidean closed cube centered at $x$ with side length $2 s$. 
First we assume that $2 \sqrt{v} s \leq t$. For any $\epsilon>0, d\left(y, t ; y^{\prime}, t\right) \leq \epsilon$ whenever $\left|y-y^{\prime}\right| \leq C_{v}^{-1} \cdot \epsilon^{4} t^{2 v-3}$. Therefore, for some $C_{v}$ with possibly different value,

$$
N(\epsilon, \overline{S(x, s)} \times\{t\}) \leq C_{v}\left(s \epsilon^{-4} t^{3-2 v}\right)^{v} .
$$

Besides, (3.10) implies that

$$
\operatorname{diam}_{d}(\overline{S(x, s)} \times\{t\}) \leq C_{v} \cdot s^{1 / 4} t^{(3-2 v) / 4} .
$$

By the standard results on entropy ([1], Theorem 1.3.3), there exists a universal constant $K>0$ (later $K$ will be absorbed by $C_{v}$ ) such that

$$
\begin{aligned}
\mathbb{E}^{\mathcal{W}}\left[\sup _{y \in \overline{S(x, s)}}\left|\bar{\theta}_{t}(y)\right|\right] & \leq K \int_{0}^{\operatorname{diam}_{d}(\overline{S(x, s)} \times\{t\}) / 2} \sqrt{\ln N(\epsilon, \overline{S(x, s)} \times\{t\})} d \epsilon \\
& \leq 4 K v \int_{0}^{C_{v} \cdot s^{1 / 4} t^{(3-2 v) / 4}} \sqrt{\ln \left(C_{v} \cdot s^{1 / 4} t^{(3-2 v) / 4} \epsilon^{-1}\right)} d \epsilon . \\
& \leq C_{v} \cdot s^{1 / 4} t^{(3-2 v) / 4} \int_{0}^{\infty} e^{-u^{2}} u^{2} d u
\end{aligned}
$$

which leads to (3.11).

Next, if $2 \sqrt{\nu} s>t$, then $\operatorname{diam}_{d}(\overline{S(x, s)} \times\{t\}) \leq C_{\nu} \cdot t^{1-\nu / 2}$. Following exactly the same arguments as earlier, we arrive at

$$
\mathbb{E}^{\mathcal{W}}\left[\sup _{y \in \overline{S(x, s)}}\left|\bar{\theta}_{t}(y)\right|\right] \leq C_{v} \cdot s^{1 / 4} t^{(3-2 v) / 4} \int_{\sqrt{\ln \left(C_{v} \cdot s^{1 / 4} t^{-1 / 4}\right)}}^{\infty} e^{-u^{2}} u^{2} d u .
$$

Combining this with the fact that

$$
\int_{a}^{\infty} e^{-u^{2}} u^{2} d u=\mathcal{O}\left(a e^{-a^{2}}\right) \text { for sufficiently large } a>0
$$

we arrive at the desired conclusion.

\subsection{When $p \geq 2$}

As shown in Lemma 3, the process given by the concentric spherical averages of the dim- $v$ order-1 GFF is a (backward) Markov process, which enables the renormalization that transforms it into a time-changed Brownian motion. However, when $(I-\Delta)$ is replaced by $(I-\Delta)^{p}$ for $p \geq 2$, spherical averages of the corresponding GFF no longer possess such properties. In particular, for the dim- $v$ order- $p$ GFF with $p \geq 2$, for any fixed $x \in \mathbb{R}^{v}$, the concentric spherical average process $\left\{\mathcal{I}\left(h_{\sigma_{x, t} \text { ave }}\right): t \in(0,1]\right\}$ fails to be (backward) Markovian. However, it is still possible to explicitly compute the covariance of this process, the result of which shows that, although not being an exact one, the process is "close" to becoming a Markov process. To make this rigorous, we adopt the same method as the one presented in [5]. For simplicity, we only outline the idea here and refer to [5] for more details.

The derivations of the covariance of the spherical averages, as shown in Section 3.1 and Section 3.2, can be generalized to the operator $m^{2}-\Delta$ for any $m>0$. To be specific, if the operator $I-\Delta$ is replaced by $m^{2}-\Delta$ in constructing the dim- $v$ order-1 GFF, then for every $x, y \in \mathbb{R}^{v}$ and every $t, s \in(0,1]$,

$$
\begin{aligned}
\mathbb{E}^{\mathcal{W}}\left[\mathcal{I}\left(h_{\sigma_{x, t}^{\text {ave }}} \mathcal{I}\left(h_{\sigma_{y, s}^{\text {ave }}}\right)\right]\right. \\
\quad=\frac{(2 \pi)^{v / 2}}{\alpha_{v}^{2}(t s|x-y|)^{\frac{v-2}{2}}} \int_{0}^{\infty} \frac{\tau^{2-\frac{v}{2}} J_{\frac{v-2}{2}}(t \tau) J_{\frac{v-2}{2}}(s \tau) J_{\frac{v-2}{2}}(|x-y| \tau)}{m^{2}+\tau^{2}} d \tau .
\end{aligned}
$$


Comparing this expression with the general formula (3.2), one can easily verify that, for the dim- $v$ order- $p$ GFF, $\mathbb{E}^{\mathcal{W}}\left[\mathcal{I}\left(h_{\sigma_{x, t}^{\text {ave }}}\right) \mathcal{I}\left(h_{\sigma_{y, s}^{\text {ave }}}\right)\right]$ is equal to

$$
\frac{(2 \pi)^{v / 2} /(p-1) !}{\alpha_{\nu}^{2}(t s|x-y|)^{\frac{v-2}{2}}}\left(\frac{-1}{2 m} \frac{d}{d m}\right)_{m=1}^{p-1}\left[\int_{0}^{\infty} \frac{\tau^{2-\frac{v}{2}} J_{\frac{v-2}{2}}(t \tau) J_{\frac{v-2}{2}}(s \tau) J_{\frac{\nu-2}{2}}(|x-y| \tau)}{m^{2}+\tau^{2}} d \tau\right] .
$$

In particular, when $x=y$ and $0<s \leq t \leq 1$,

$$
\begin{aligned}
\mathbb{E}^{\mathcal{W}}\left[\mathcal{I}\left(h_{\sigma_{x, t}^{\text {ave }}}\right) \mathcal{I}\left(h_{\sigma_{x, s}^{\text {ave }}}\right)\right] & \\
= & \frac{1}{\alpha_{\nu}(t s)^{\frac{\nu-2}{2}}(p-1) !}\left(\frac{-1}{2 m} \frac{d}{d m}\right)_{m=1}^{p-1}\left[K_{\frac{\nu-2}{2}}(m t) I_{\frac{\nu-2}{2}}(m s)\right],
\end{aligned}
$$

which obviously takes the form of

$$
\sum_{k=1}^{p} a_{k}(t) b_{k}(s)
$$

where functions $a_{k}$ only depend on $t$ and functions $b_{k}$ only depend on $s$ for each $k=1, \ldots, p$. A covariance of the form of (3.12) does indicate that the Gaussian process $\left\{\mathcal{I}\left(h_{\sigma_{x, t}^{\text {ave }}}\right): t \in(0,1]\right\}$ is not (backward) Markovian. Heuristically speaking, at any given radius, the spherical average alone "provides" too little information for one to predict how the process will evolve for smaller radii. To restore the Markov property, we "collect" more information ${ }^{10}$ about the GFF over each sphere.

To this end, recall the remark at the end of Section 2 that the higher the order of the operator is, the more regular the corresponding GFF becomes. In particular, for $p \geq 2$, the $l$ th derivative of the spherical average measure in radius, i.e., $(d / d t)^{l} \sigma_{x, t}^{\text {ave }}$ in the sense of tempered distribution, also gives rise to a Paley-Wiener integral $\mathcal{I}\left(h_{\left.(d / d t)^{l} \sigma_{x, t}^{\text {ave }}\right)}\right.$ for $l=1, \ldots, p-1$. It turns out that, with $x \in \mathbb{R}^{v}$ fixed, if $\mathbf{V}_{x, t}$, for $t \in(0,1]$, is the $\mathbb{R}^{p}$-valued random variable on $\Theta^{p}$ given by

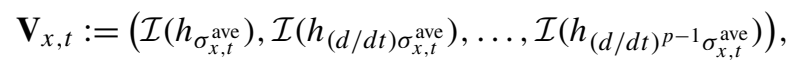

then the process $\left\{\mathbf{V}_{x, t}: t \in(0,1]\right\}$ is a $\mathbb{R}^{p}$-valued Gaussian (backward) Markov process, and for $0<s \leq t \leq 1$,

$$
\mathbb{E}^{\mathcal{W}^{p}}\left[\left(\mathbf{V}_{x, t}\right)^{\top}\left(\mathbf{V}_{x, s}\right)\right]=\mathbf{A}(t) \cdot \mathbf{B}(s),
$$

where "." here refers to matrix multiplication, $\mathbf{A}(t)$ and $\mathbf{B}(s)$ are two $p \times p$ matrices depending only on $t$ and, respectively, only on $s$, and for $1 \leq i, j \leq p$,

$$
(\mathbf{A}(t))_{i j}=\left(\frac{d}{d t}\right)^{i-1} a_{j}(t) \text { and }(\mathbf{B}(s))_{i j}=\left(\frac{d}{d s}\right)^{j-1} b_{i}(s),
$$

where $a_{j}$ 's and $b_{i}$ 's are as in (3.12). In other words, when collecting simultaneously the spherical average and its first $(p-1)$ st order derivatives, the Markov property is restored by this vector-valued process. Furthermore, the matrix $\mathbf{B}(s)$ is non-degenerate when $s$ is sufficiently small, so (3.13) also leads to a renormalization which is $\mathbf{U}_{x, t}:=\mathbf{V}_{x, t} \cdot \mathbf{B}^{-1}(t)$. It follows from (3.13) that, for $0<s \leq t \leq 1$,

$$
\mathbb{E}^{\mathcal{W} \mathcal{W}^{p}}\left[\left(\mathbf{U}_{x, t}\right)^{\top}\left(\mathbf{U}_{x, s}\right)\right]=\mathbf{B}^{-1}(t) \cdot \mathbf{A}(t) .
$$

The renormalized process $\left\{\mathbf{U}_{x, t}: t \in(0,1]\right\}$ has independent increment (in the direction of $t$ decreasing). Moreover, it is possible to find a constant vector $\xi \in \mathbb{R}^{p}$ such that, as $t \searrow 0$,

$$
\left(\sigma_{x, t}^{\text {ave }},(d / d t) \sigma_{x, t}^{\text {ave }}, \ldots,(d / d t)^{p-1} \sigma_{x, t}^{\text {ave }}\right) \cdot \mathbf{B}^{-1}(t) \cdot \xi^{\top} \rightarrow \delta_{x}
$$

\footnotetext{
${ }^{10}$ This idea was originally proposed by D. Stroock during a discussion with the author.
} 
in the sense of tempered distribution; this is because the coefficient of $(d / d t)^{l} \sigma_{x, t}^{\text {ave }}$, as a function of $t$, decays sufficiently fast as $t \searrow 0$ for each $l=1, \ldots, p-1$. Therefore, $\bar{\theta}_{t}(x):=\mathbf{U}_{x, t}(\theta) \cdot \xi^{\top}$ still is a legitimate approximation of " $\theta(x)$ " when $t$ is small. In other words, although the derivatives of the spherical averages are introduced to recover the Markov property, these derivatives do not affect the approximation of pointwise values of the GFF. Moreover, the two-parameter family $\left\{\bar{\theta}_{t}(x): x \in \mathbb{R}^{v}, t \in(0,1]\right\}$ possesses the same properties as those shown in Section 3.1 and Section 3.2.

Governed by the Green's function of $(I-\Delta)^{p}$ on $\mathbb{R}^{v}$, the dim- $v$ order- $p$ GFF is polynomially correlated with the degree of the polynomial being $v-2 p$. In fact, later discussions in this article, i.e., the study of thick point, only requires the existence of an approximation $\bar{\theta}_{t}(x)$ such as the one obtained above. Therefore, it is sufficient to assume $p=1$ and investigate the thick point problem for the dim- $v$ order-1 GFF with arbitrary $v>2$.

\section{Thick points of Gaussian free fields}

To study the thick points of the dim-v order-1 GFF $(H, \Theta, \mathcal{W})$, the first problem we face is to determine a proper definition for the notion of "thick point". On one hand, inspired by (1.1) the thick point definition of log-correlated GFFs, we want to investigate the points $x \in \overline{S(O, 1)}$ where the rate of $\bar{\theta}_{t}(x)$ "blowing up" as $t \searrow 0$ is comparable with certain function in $t$ that is singular at $t=0$. On the other hand, a polynomial-correlated GFF is expected to behave differently from a log-correlated GFF in various aspects. Firstly, for each fixed $x \in \overline{S(O, 1)}$, as $t$ decays, the Gaussian process $\left\{\bar{\theta}_{t}(x): t \in(0,1]\right\}$ has a variance that grows faster than in the log-correlated case, which makes $\bar{\theta}_{t}(x)$ more unstable and less likely to achieve an "unusually" large value through a limit such as the one in (1.1). Secondly, for any $x, y \in \overline{S(O, 1)}$ and $x \neq y, \operatorname{Cov}\left(\bar{\theta}_{t}(x), \bar{\theta}_{s}(y)\right)=\mathcal{O}\left(|x-y|^{2-v}\right)$ for sufficiently small $t$ and $s$, so for $x$ and $y$ being close, the covariance is stronger than in the log-correlated case, which makes thick points, defined in any reasonable sense, tend to form a sparse subset of $\overline{S(O, 1)}$. Both factors contribute to making thick points of a polynomial-correlated GFF harder to detect. Taking into account of these considerations, we adopt a thick point definition that is different from (1.1) but proven to be more suitable for polynomial-correlated GFFs.

Definition 8. Let $\gamma \geq 0$. For each $\theta \in \Theta, x \in \overline{S(O, 1)}$ is a $\gamma$-thick point of $\theta$ if

$$
\limsup _{t \searrow 0} \frac{\bar{\theta}_{t}(x)}{\sqrt{-G(t) \ln t}} \geq \sqrt{2 v \gamma},
$$

where $G(t):=\mathbb{E}^{\mathcal{W}}\left[\left(\bar{\theta}_{t}(x)\right)^{2}\right]$.

We denote by $T_{\theta}^{\gamma}$ the set of all the $\gamma$-thick points of $\theta$. Since $\bar{\theta}_{t}(x)$ is assumed to be continuous in $(x, t) \in \mathbb{R}^{v} \times(0,1]$, $T_{\theta}^{\gamma}$ is a measurable subset of $\overline{S(O, 1)}$. Moreover, viewing from the perspective of (3.6), if $\gamma>0$, (4.1) requires $\bar{\theta}_{t}(x)$ to grow, as $t \searrow 0$, no slower than a unusually large function in $t$, at least along a sequence in $t$. In this sense, the value of $\theta$ is unusually large at a $\gamma$-thick point so long as $\gamma>0$. Compared with (1.1), the requirement in (4.1) is easier to achieve, which contributes positively to $T_{\theta}^{\gamma}$ having "detectable" mass. In fact, such a deviation from (1.1) (i.e., replacing in the definition " $\lim _{t \searrow 0}$ " by "lim $\sup _{t \searrow 0}$ " and "=" by " $\geq$ ") is necessary, as we will see later in Section 4.2.

Our main goal is to determine the Hausdorff dimension of $T_{\theta}^{\gamma}$, denoted by $\operatorname{dim}_{\mathcal{H}}\left(T_{\theta}^{\gamma}\right)$. We state the main result below.

Theorem 9. For $\gamma \in[0,1]$,

$$
\operatorname{dim}_{\mathcal{H}}\left(T_{\theta}^{\gamma}\right)=v(1-\gamma) \quad \text { a.s. }
$$

Moreover, for $\mathcal{W}$-a.e. $\theta \in \Theta, x \in T_{\theta}^{0}$ for Lebesgue-a.e. $x \in \overline{S(O, 1)}$, and $T_{\theta}^{\gamma}=\varnothing$ when $\gamma>1$.

The theorem is proven by establishing the upper bound and the lower bound separately. More specifically, we prove in Section 4.1

$$
\text { the upper bound: } \operatorname{dim}_{\mathcal{H}}\left(T_{\theta}^{\gamma}\right) \leq v(1-\gamma) \text { a.s. }
$$


Then we devote the entire Section 5 to proving

$$
\text { the lower bound: } \operatorname{dim}_{\mathcal{H}}\left(T_{\theta}^{\gamma}\right) \geq v(1-\gamma) \text { a.s. }
$$

As mentioned earlier, the polynomially singular covariance of the GFF makes thick points rare and hence hard to detect, as a consequence of which, the upper bound on the Hausdorff dimension of $T_{\theta}^{\gamma}$ is readily obtained, but deriving the lower bound is more complicated.

\subsection{Proof of the upper bound}

The derivation of the upper bound (4.2) follows an adaptation of the procedure used in [17]. The idea behind of the proof is that, if $x$ is a thick point of $\theta$, i.e., if $\bar{\theta}_{t}(x)$ achieves "unusually" large values, then based on the continuity results of the family $\left\{\bar{\theta}_{t}(x):(x, t) \in \overline{S(O, 1)} \times(0,1]\right\}$ established in Section 3, one can argue that, whenever $(y, r)$ is sufficiently close to $(x, t), \bar{\theta}_{r}(y)$ will also be "unusually" large. This enables us to reduce the problem to the study of a discrete Gaussian family, i.e., the family of $\bar{\theta}_{r}(y)$ 's for $(y, r)$ chosen from a lattice approximation of $\overline{S(O, 1)} \times(0,1]$. First, we will apply the results in Section 3 to show that, at least for our purpose, to study the behavior of $\bar{\theta}_{t}(x)$, it does no harm to restrict $(x, t)$ on a lattice approximation of $\overline{S(O, 1)} \times(0,1]$. To simplify the notation, we write

$$
D(t):=\sqrt{-G(t) \ln t} \text { for } t \in(0,1] .
$$

Lemma 10. There exists a constant $C_{v}>0$ such that for every $x \in \overline{S(O, 1)}$ and every $n \geq 1$,

$$
\mathbb{E}^{\mathcal{W}}\left[\sup _{(y, t) \in \overline{S\left(x, 2^{-n}\right)} \times\left[2^{-n}, 2^{-n+1}\right]} \frac{\bar{\theta}_{t}(y)}{D(t)}\right] \leq C_{\nu} \cdot \frac{1}{\sqrt{n}} .
$$

Proof. Similarly as in Lemma 7, we will prove the desired result by the metric entropy method. Let $n \geq 1$ be fixed. For every $\epsilon>0$, set

$$
\tau_{n, \epsilon}:=\frac{1}{2}\left[\left(\frac{\epsilon^{2}}{9} \cdot C_{v}^{-1} \cdot 2^{-(n+1)(v-3 / 2)}\right)^{2} \wedge 2^{-n-1}\right],
$$

where $C_{v}$, for the moment, is the same constant as in (3.10). Let

$$
\left\{B\left(y_{l}, \tau_{n, \epsilon}\right): l=1, \ldots, L_{\epsilon}\right\}
$$

be a finite covering of $\overline{S\left(x, 2^{-n}\right)}$ where $y_{l} \in S\left(x, 2^{-n}\right)$ and $L_{\epsilon}$ is the smallest number of balls $B\left(y_{l}, \tau_{n, \epsilon}\right)$ needed to cover $\overline{S\left(x, 2^{-n}\right)}$ and hence $L_{\epsilon}=\mathcal{O}\left(2^{-n v} / \tau_{n, \epsilon}^{v}\right)$. By (3.10), the choice of $\tau_{n, \epsilon}$ is such that the diameter of each ball $B\left(y_{l}, \tau_{n, \epsilon}\right)$ under the metric $d\left(\cdot, 2^{-n-1} ; *, 2^{-n-1}\right)$ is no greater than $\epsilon / 3$. In fact, for any $t \geq 2^{-n-1}$, (3.10) implies that, if $\left|y-y^{\prime}\right| \leq 2 \tau_{n, \epsilon}$, then

$$
d^{2}\left(y, t ; y^{\prime}, t\right) \leq C_{\nu} t^{3 / 2-v} \sqrt{2 \tau_{n, \epsilon}} \leq \epsilon^{2} / 9 .
$$

Next, take $\tau_{0}:=2^{-n-1}$ and define $\tau_{m}$ inductively such that

$$
G\left(\tau_{m-1}\right)-G\left(\tau_{m}\right)=\epsilon^{2} / 9
$$

for $m=1, \ldots, M_{\epsilon}$, where $M_{\epsilon}$ is the smallest integer such that $\tau_{M_{\epsilon}} \geq 2^{-n+2}$ and hence

$$
M_{\epsilon}=\mathcal{O}\left(G\left(2^{-n}\right)\right) / \epsilon^{2} \text {. }
$$

Consider the covering of $\overline{S\left(x, 2^{-n}\right)} \times\left[2^{-n}, 2^{-n+1}\right]$ that consists of the cylinders

$$
\left\{B\left(y_{l}, \tau_{n, \epsilon}\right) \times\left(\tau_{m-1}, \tau_{m}\right): l=1, \ldots, L_{\epsilon}, m=1, \ldots, M_{\epsilon}\right\} .
$$


Any pair of points $((y, t),(w, s))$ that lies in one of the cylinders above, e.g., $B\left(y_{l}, \tau_{n, \epsilon}\right) \times\left(\tau_{m-1}, \tau_{m}\right)$, satisfies that

$$
\begin{aligned}
d(y, t ; w, s) & \leq d\left(y, t ; y, \tau_{m}\right)+d\left(y, \tau_{m} ; w, \tau_{m}\right)+d\left(w, \tau_{m} ; w, s\right) \\
& \leq \epsilon / 3+\epsilon / 3+\epsilon / 3=\epsilon .
\end{aligned}
$$

This implies that

$$
N\left(\epsilon, \overline{S\left(x, 2^{-n}\right)} \times\left[2^{-n}, 2^{-n+1}\right]\right) \leq L_{\epsilon} \cdot M_{\epsilon},
$$

where $N$ is the entropy function defined before Lemma 7. Moreover, the diameter of $\overline{S\left(x, 2^{-n}\right)} \times\left[2^{-n}, 2^{-n+1}\right]$ under the metric $d$ is bounded by $2 \sqrt{G\left(2^{-n}\right)}$. Therefore, there is a universal constant $K>0$ (later $K$ is absorbed into $C_{v}$ ) such that

$$
\begin{aligned}
& \mathbb{E}^{\mathcal{W}}\left[\sup _{(y, t) \in \overline{S\left(x, 2^{-n}\right) \times\left[2^{-n}, 2^{-n+1}\right]}} \bar{\theta}_{t}(y)\right] \leq K \int_{0}^{\sqrt{G\left(2^{-n}\right)}} \sqrt{\ln \left(L_{\epsilon} \cdot M_{\epsilon}\right)} d \epsilon \\
& \leq K \int_{0}^{\sqrt{G\left(2^{-n}\right)}}\left(\sqrt{\ln L_{\epsilon}}+\sqrt{\ln M_{\epsilon}}\right) d \epsilon \\
& \leq C_{v} \sqrt{G\left(2^{-n}\right)} .
\end{aligned}
$$

Equation (4.4) follows from dividing both sides of the inequality above by $D\left(2^{-n+1}\right)$.

Now we can get to the proof of the upper bound (4.2).

Proof of the upper bound: When $\gamma=0,(4.2)$ is trivially satisfied. Without loss of generality, we assume that $\gamma \in(0,1]$ for the rest of the proof. For each $n \geq 0$, consider a finite lattice partition of $\overline{S(O, 1)}$ with cell size $2 \cdot 2^{-n}$ (i.e., the length, under the Euclidean metric, of each side of the cell is $\left.2 \cdot 2^{-n}\right)$. Denote $\left\{x_{j}^{(n)}: j=1, \ldots, J_{n}\right\}$ the lattice cell centers where $J_{n}=2^{v n}$ is the total number of the cells. Let $\gamma^{\prime \prime}, \gamma^{\prime}$ be two numbers such that $0<\gamma^{\prime \prime}<\gamma^{\prime}<\gamma$ and $\gamma^{\prime}$ and $\gamma^{\prime \prime}$ can be arbitrarily close to $\gamma$. Consider the subset of the indices

$$
I_{n}:=\left\{j: 1 \leq j \leq J_{n} \text { s.t. } \sup _{(y, r) \in S\left(x_{j}^{(n)}, 2^{-n}\right) \times\left[2^{-n}, 2^{-n+1}\right]} \frac{\bar{\theta}_{r}(y)}{D(r)}>\sqrt{2 \nu \gamma^{\prime}}\right\} .
$$

Combining (4.4) and the Borell-TIS inequality ([1] Section 2.1 and the references therein), we have that, for every $j=1, \ldots, J_{n}$,

$$
\begin{aligned}
\mathcal{W}\left(j \in I_{n}\right) & =\mathcal{W}\left(\sup _{(y, r) \in S\left(x_{j}^{(n)}, 2^{-n}\right) \times\left[2^{-n}, 2^{-n+1}\right]} \frac{\bar{\theta}_{r}(y)}{D(r)}>\sqrt{2 v \gamma^{\prime}}\right) \\
& \leq \exp \left[-\frac{1}{2}\left(\sqrt{2 v \gamma^{\prime}}-\frac{C_{v}}{\sqrt{n}}\right)^{2} \cdot \ln 2 \cdot(n-1)\right] \\
& \leq \exp \left(-v \gamma^{\prime \prime} \cdot \ln 2 \cdot n\right) .
\end{aligned}
$$

Therefore,

$$
\mathbb{E}^{\mathcal{W}}\left[\operatorname{card}\left(I_{n}\right)\right]=\sum_{j \in J_{n}} \mathcal{W}\left(j \in I_{n}\right) \leq C_{v} \cdot 2^{\nu\left(1-\gamma^{\prime \prime}\right) n} .
$$

On the other hand, if $y \in T_{\theta}^{\gamma}$, then there exists a sequence $\left\{t_{k}: k \geq 0\right\}$ with $t_{k} \searrow 0$ as $k \nearrow \infty$ such that

$$
\frac{\bar{\theta}_{t_{k}}(y)}{D\left(t_{k}\right)}>\sqrt{2 v \gamma^{\prime}} \quad \text { for all } k \geq 0 .
$$


For every $k$, let $n(k)$ be the unique positive integer such that

$$
2^{-n(k)} \leq t_{k}<2^{-n(k)+1} .
$$

If $x_{j}^{(n(k))}$ is the cell center (at $n(k)$ th level) such that $\left|y-x_{j}^{(n(k))}\right| \leq \sqrt{v} 2^{-n(k)}$, then clearly $j \in I_{n(k)}$. Therefore,

$$
T_{\theta}^{\gamma} \subseteq \bigcap_{m \geq 1} \bigcup_{n \geq m} \bigcup_{j \in I_{n}} \overline{S\left(x_{j}^{(n)}, 2^{-n}\right)} .
$$

Moreover, for each $m \geq 1,\left\{\overline{S\left(x_{j}^{(n)}, 2^{-n}\right)}: j \in I_{n}, n \geq m\right\}$ forms a covering of $T_{\theta}^{\gamma}$, and the diameter (under the Euclidean metric) of the member $\overline{S\left(x_{j}^{(n)}, 2^{-n}\right)}$ is $2 \sqrt{v} \cdot 2^{-n}$. Thus, if $\mathcal{H}^{\alpha}$ is the Hausdorff- $\alpha$ measure for $\alpha>0$, then

$$
\begin{aligned}
\mathcal{H}^{\alpha}\left(T_{\theta}^{\gamma}\right) & \leq \liminf _{m \rightarrow \infty} \sum_{n \geq m} \sum_{j \in I_{n}}\left(2 \sqrt{v} \cdot 2^{-n}\right)^{\alpha} \\
& =C_{\nu} \cdot \liminf _{m \rightarrow \infty} \sum_{n \geq m} \operatorname{card}\left(I_{n}\right) 2^{-n \alpha} .
\end{aligned}
$$

It follows from Fatou's lemma that

$$
\begin{aligned}
\mathbb{E}^{\mathcal{W}}\left[\mathcal{H}^{\alpha}\left(T_{\theta}^{\gamma}\right)\right] & \leq C_{v} \cdot \liminf _{m \rightarrow \infty} \sum_{n \geq m} \mathbb{E}^{\mathcal{W}}\left[\operatorname{card}\left(I_{n}\right)\right] \cdot 2^{-n \alpha} \\
& \leq C_{v} \cdot \lim _{m \rightarrow \infty} \sum_{n \geq m} 2^{\left[v\left(1-\gamma^{\prime \prime}\right)-\alpha\right] n}
\end{aligned}
$$

Clearly, for any $\alpha>v\left(1-\gamma^{\prime \prime}\right)$, with probability one, $\mathcal{H}^{\alpha}\left(T_{\theta}^{\gamma}\right)=0$ and hence $\operatorname{dim}_{\mathcal{H}}\left(T_{\theta}^{\gamma}\right) \leq \alpha$. Since $\gamma^{\prime \prime}$ is arbitrarily close to $\gamma$, we conclude that

$$
\operatorname{dim}_{\mathcal{H}}\left(T_{\theta}^{\gamma}\right) \leq v(1-\gamma) \text { a.s. }
$$

We have completed the proof of the upper bound (4.2). In addition, following the same arguments as above, if $\gamma>1$, we can choose $\gamma^{\prime \prime}$ to be greater than 1 , in which case

$$
\sum_{n \geq m} \mathbb{E}^{\mathcal{W}}\left[\operatorname{card}\left(I_{n}\right)\right] \leq \sum_{n \geq m} 2^{v\left(1-\gamma^{\prime \prime}\right) n} \rightarrow 0 \quad \text { as } m \rightarrow \infty
$$

This observation immediately implies the last assertion in Theorem 9, that is, if $\gamma>1$, then $T_{\theta}^{\gamma}=\varnothing$ a.s.

\subsection{Perfect $\gamma$-thick point}

In this subsection we explain why the definition (4.1) is more proper for the study of thick points for polynomialcorrelated GFFs. Simply speaking, the straightforward analogue of (1.1), the thick point definition for log-correlated GFFs, imposes too strong a condition to fulfill in the case of polynomial-correlated GFFs. To make this precise, we first define a more strict analogue of (1.1).

Definition 11. Let $\gamma \geq 0$. For each $\theta \in \Theta, x \in \overline{S(O, 1)}$ is called a perfect $\gamma$-thick point of $\theta$ if

$$
\lim _{t \searrow 0} \frac{\bar{\theta}_{t}(x)}{\sqrt{-G(t) \ln t}}=\sqrt{2 \nu \gamma} .
$$

Again, if $P T_{\theta}^{\gamma}$ is the set that contains all the perfect $\gamma$-thick points of $\theta$, then $P T_{\theta}^{\gamma}$ is a measurable subset of $\overline{S(O, 1)}$. This subsection will be devoted to showing that if $\gamma>0$, then perfect $\gamma$-thick point does not exist, i.e, $P T_{\theta}^{\gamma}=\varnothing$, for 
$\mathcal{W}$-a.e. $\theta \in \Theta$. Recall that $D(t)=\sqrt{-G(t) \ln t}$. Compared with (4.1), the definition given above imposes a stronger condition because the existence of the limit in (4.5) requires that $\bar{\theta}_{t}(x)-\sqrt{2 v \gamma} D(t)=o(D(t))$ for all sufficiently small $t$. However, we will see that the probability of $\bar{\theta}_{t}(x)$ exhibiting such stable behaviors decays very fast as $t$ tends to zero; this is not surprising because, according to Remark $4, \bar{\theta}_{t}(x)$ can be viewed as a Brownian motion $B_{\tau}$ running by the clock $\tau=G(t)$ which blows up very fast as $t \searrow 0$, and hence one would expect $\bar{\theta}_{t}(x)$ to be rather unstable as $t$ varies. To make these arguments rigorous, we follow a similar strategy as in Section 4.1, which is to first use the continuity properties of $\bar{\theta}_{t}(x)$ to reduce the problem to its discrete approximation.

For each $n \geq 0$, set $s_{n}:=2^{-n^{2}}$. For $n \geq 1$, consider the two-parameter Gaussian family

$$
\mathcal{A}_{n}:=\left\{\bar{\theta}_{t}(x): x \in \overline{S(O, 1)}, t \in\left[s_{n}, s_{n-1}\right]\right\} .
$$

Let $\omega_{n}$ be the modulus of continuity of $\mathcal{A}_{n}$ under the intrinsic metric $d$, i.e., for every $\delta>0$,

$$
\omega_{n}(\delta):=\sup \left\{\left|\bar{\theta}_{t}(x)-\bar{\theta}_{s}(y)\right|: d(x, t ; y, s) \leq \delta, x, y \in \overline{S(O, 1)}, t, s \in\left[s_{n}, s_{n-1}\right]\right\} .
$$

Lemma 12. There exists a constant $C_{v}>0$ such that for every $n \geq 1$ and every $0<\delta \ll \sqrt{G\left(s_{n}\right)}$,

$$
\mathbb{E}^{\mathcal{W}}\left[\omega_{n}(\delta)\right] \leq C_{v} \cdot \delta \sqrt{\ln \left(s_{n}^{(3-2 v) / 4} / \delta\right)}
$$

Moreover, if

$$
\mathcal{B}_{n}:=\left\{(x, y) \in(\overline{S(O, 1)})^{2}:|x-y| \leq \sqrt{v} \cdot s_{2 n}\right\},
$$

then

$$
\mathcal{W}\left(\sup _{(x, y) \in \mathcal{B}_{n}, t \in\left[s_{n}, s_{n-1}\right]}\left|\frac{\bar{\theta}_{t}(x)}{D(t)}-\frac{\bar{\theta}_{t}(y)}{D(t)}\right|>2^{-3 n^{2} / 16}, \text { i.o. }\right)=0 .
$$

Proof. For every $\epsilon>0$, let $N\left(\epsilon, \mathcal{A}_{n}\right)$ be the entropy function, as introduced before Lemma 7 . Then, it follows from a similar argument as the one used in the proof of Lemma 10 that $N\left(\epsilon, \mathcal{A}_{n}\right) \leq L_{\epsilon} \cdot M_{\epsilon}$ where $L_{\epsilon}=\mathcal{O}\left(s_{n}^{\nu(3-2 v)}\right) / \epsilon^{4 v}$ is the entropy for the set $\overline{S(O, 1)}$ under the metric $d\left(\cdot, s_{n} ; *, s_{n}\right)$, and $M_{\epsilon}=\mathcal{O}\left(G\left(s_{n}\right)\right) / \epsilon^{2}$ is the entropy for the interval $\left[s_{n}, s_{n-1}\right]$ corresponding to the concentric process $\left\{\bar{\theta}_{t}(x): t \in\left[s_{n}, s_{n-1}\right]\right\}$ for any fixed $x$. Hence ([1], Corollary 1.3.4), there exists a universal constant $K>0$ such that for every $n \geq 1$,

$$
\mathbb{E}^{\mathcal{W}}\left[\omega_{n}(\delta)\right] \leq K \int_{0}^{\delta} \sqrt{\ln N\left(\epsilon, \mathcal{A}_{n}\right)} d \epsilon \leq K \int_{0}^{\delta}\left(\sqrt{\ln L_{\epsilon}}+\sqrt{\ln M_{\epsilon}}\right) d \epsilon .
$$

Similarly as the integrals we evaluated when proving (3.11), we have that

$$
\int_{0}^{\delta} \sqrt{\ln L_{\epsilon}} d \epsilon \leq C_{v} \cdot \delta \sqrt{\ln \left(s_{n}^{(3-2 v) / 4} / \delta\right)}
$$

and

$$
\int_{0}^{\delta} \sqrt{\ln M_{\epsilon}} d \epsilon \leq C_{v} \cdot \delta \sqrt{\ln \left(s_{n}^{(1-v / 2)} / \delta\right)}
$$

for some $C_{v}>0$, which lead to the first assertion.

To prove the second assertion, notice that by (3.10), if $|x-y| \leq \sqrt{v} \cdot s_{2 n}$, then for any $t \in\left[s_{n}, s_{n-1}\right]$,

$$
d(x, t ; y, t) \leq C_{v} \cdot t^{(3-2 v) / 4} \cdot s_{2 n}^{1 / 4} \leq C_{v} \cdot 2^{n^{2}(2 v-7) / 4} .
$$


Therefore, by (4.6),

$$
\begin{aligned}
& \mathbb{E}^{\mathcal{W}}\left[\sup _{(x, y) \in \mathcal{B}_{n}, t \in\left[s_{n}, s_{n-1}\right]}\left|\bar{\theta}_{t}(x)-\bar{\theta}_{t}(y)\right|\right] \leq \mathbb{E}^{\mathcal{W}}\left[\omega_{n}\left(C_{v} \cdot 2^{n^{2}(v / 2-7 / 4)}\right)\right] \\
& \quad \leq C_{v} \cdot 2^{n^{2}(v / 2-7 / 4)} \cdot n .
\end{aligned}
$$

The desired conclusion follows from dividing both sides of the inequality above by $D\left(s_{n-1}\right)$ and applying the BorelCantelli lemma.

Now we are ready to establish the main result of this subsection, that is, if $\gamma>0$, then the perfect $\gamma$-thick point does not exist almost surely. Being "perfect" prevents such points from existing.

Theorem 13. If $\gamma>0$, then $P T_{\theta}^{\gamma}=\varnothing$ a.s.

Proof. Based on (4.7), for $\mathcal{W}$-a.e. $\theta$, there exists $N_{\theta} \in \mathbb{N}$ such that for every $n \geq N_{\theta}$ and $x, y$ such that $y \in \overline{S\left(x, s_{2 n}\right)}$,

$$
\sup _{t \in\left[s_{n}, s_{n-1}\right]}\left|\frac{\bar{\theta}_{t}(x)}{D(t)}-\frac{\bar{\theta}_{t}(y)}{D(t)}\right| \leq 2^{-3 n^{2} / 16} .
$$

Choose $M>0$ to be a sufficiently large number. Consider the lattice partition of $\overline{S(O, 1)}$ with cell size $2^{-k}$, and let

$$
\left\{x_{j}^{(k)}: j=1, \ldots, J_{k}\right\}
$$

be the cell centers. Let $y$ be a perfect $\gamma$-thick point. For $n$ that is sufficiently large, if $x_{j}^{\left(4 n^{2}\right)}$ is the cell center such that $y \in \overline{S\left(x_{j}^{\left(4 n^{2}\right)}, s_{2 n}\right)}$, then

$$
\sup _{t \in\left[s_{n}, s_{n-1}\right]}\left|\frac{\bar{\theta}_{t}\left(x_{j}^{\left(4 n^{2}\right)}\right)}{D(t)}-\sqrt{2 v \gamma}\right| \leq \frac{1}{M} .
$$

In particular, this means that

$$
\left|\bar{\theta}_{s_{n-1}}\left(x_{j}^{\left(4 n^{2}\right)}\right)-\sqrt{2 v \gamma} D\left(s_{n-1}\right)\right| \leq \frac{D\left(s_{n-1}\right)}{M},
$$

and for every $t \in\left[s_{n}, s_{n-1}\right]$,

$$
\left|\bar{\theta}_{t}\left(x_{j}^{\left(4 n^{2}\right)}\right)-\bar{\theta}_{s_{n-1}}\left(x_{j}^{\left(4 n^{2}\right)}\right)-\sqrt{2 v \gamma}\left[D(t)-D\left(s_{n-1}\right)\right]\right| \leq \frac{2 D(t)}{M} .
$$

Let $\mathcal{P}_{n}=\mathcal{P}_{n}^{x_{j}^{\left(4 n^{2}\right)}} \subseteq \Theta$ be the collection of all the field elements $\theta$ such that

$$
\forall t \in\left[s_{n}, s_{n-1}\right], \quad\left|\bar{\theta}_{t}\left(x_{j}^{\left(4 n^{2}\right)}\right)-\bar{\theta}_{s_{n-1}}\left(x_{j}^{\left(4 n^{2}\right)}\right)-\sqrt{2 v \gamma}\left[D(t)-D\left(s_{n-1}\right)\right]\right| \leq \frac{2 D(t)}{M} .
$$

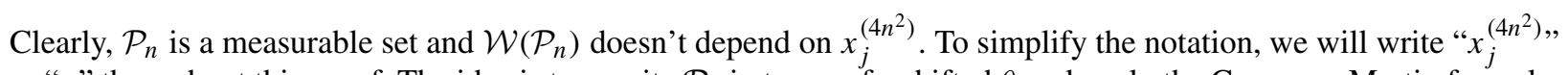
as " $x$ " throughout this proof. The idea is to rewrite $\mathcal{P}_{n}$ in terms of a shifted $\theta$ and apply the Cameron-Martin formula. To this end, we define, for $t \in(0,1]$,

$$
F(t):=\frac{D^{\prime}(t)}{G^{\prime}(t)} \text { and } \quad f(t):=F^{\prime}(t)
$$


and let $\zeta_{x, n}$ be the element in $H^{-1}\left(\mathbb{R}^{v}\right)$ such that its corresponding Paley-Wiener integral is

$$
\mathcal{I}\left(h_{\zeta_{x, n}}\right)(\theta)=\int_{s_{n}}^{s_{n-1}}\left(\bar{\theta}_{t}(x)-\bar{\theta}_{s_{n-1}}(x)\right) f(t) d t+F\left(s_{n}\right)\left(\bar{\theta}_{s_{n}}(x)-\bar{\theta}_{s_{n-1}}(x)\right) .
$$

We observe that for every $t \in\left[s_{n}, s_{n-1}\right]$,

$$
\begin{aligned}
\left(h_{\bar{\sigma}_{x, t}}, h_{\zeta x, n}\right)_{H}= & \int_{s_{n}}^{t}\left[G(t)-G\left(s_{n-1}\right)\right] f(s) d s+\int_{t}^{s_{n-1}}\left[G(s)-G\left(s_{n-1}\right)\right] f(s) d s \\
& +F\left(s_{n}\right)\left[G(t)-G\left(s_{n-1}\right)\right] \\
= & {\left[G(t)-G\left(s_{n-1}\right)\right] F(t)+\int_{t}^{s_{n-1}}\left[G(s)-G\left(s_{n-1}\right)\right] f(s) d s } \\
= & -\int_{t}^{s_{n-1}} G^{\prime}(s) F(s) d s=-\int_{t}^{s_{n-1}} D^{\prime}(s) d s \\
= & D(t)-D\left(s_{n-1}\right) .
\end{aligned}
$$

Therefore,

$$
\mathcal{I}\left(h_{\bar{\sigma}_{x, t}}-h_{\bar{\sigma}_{x, s_{n-1}}}\right)\left(\theta-\sqrt{2 v \gamma} h_{\zeta_{x, n}}\right)=\bar{\theta}_{t}(x)-\bar{\theta}_{s_{n-1}}(x)-\sqrt{2 v \gamma}\left[D(t)-D\left(s_{n-1}\right)\right] .
$$

In other words, we can view

$$
\bar{\theta}_{t}(x)-\bar{\theta}_{s_{n-1}}(x)-\sqrt{2 \nu \gamma}\left[D(t)-D\left(s_{n-1}\right)\right]
$$

as a Paley-Wiener integral of a translated GFF. Thus, by the Cameron-Martin formula ([25], Theorem 8.2.9), $\mathcal{W}\left(\mathcal{P}_{n}\right)$ is equal to

$$
\begin{aligned}
& \mathbb{E}^{\mathcal{W}}\left[e^{-\sqrt{2 v \gamma} \mathcal{I}\left(h_{\zeta x, n}\right)-v \gamma\left\|h_{\zeta x, n}\right\|_{H}^{2}} ; \forall t \in\left[s_{n}, s_{n-1}\right],\left|\bar{\theta}_{t}(x)-\bar{\theta}_{s_{n-1}}(x)\right| \leq \frac{2 D(t)}{M}\right]
\end{aligned}
$$

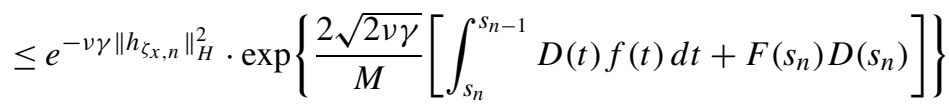

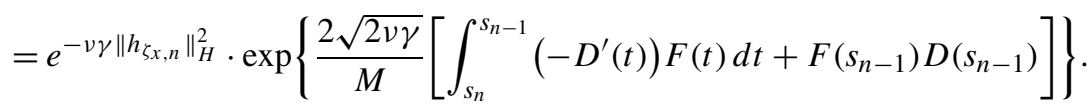

Moreover, we compute

$$
\begin{aligned}
\left\|h_{\zeta_{x, n}}\right\|_{H}^{2} & =\int_{s_{n}}^{s_{n-1}}\left[D(t)-D\left(s_{n-1}\right)\right] f(t) d t+F\left(s_{n}\right)\left[D\left(s_{n}\right)-D\left(s_{n-1}\right)\right] \\
& =\int_{s_{n}}^{s_{n-1}}\left(-D^{\prime}(t)\right) F(t) d t .
\end{aligned}
$$

It is easy to verify that, when $n$ is large,

$$
\int_{s_{n}}^{s_{n-1}}\left(-D^{\prime}(t)\right) F(t) d t=\mathcal{O}\left(n^{3}\right) \quad \text { and } \quad F\left(s_{n-1}\right) D\left(s_{n-1}\right)=\mathcal{O}\left(n^{2}\right) .
$$

Thus, $\mathcal{W}\left(\mathcal{P}_{n}\right)$ is no greater than

$$
\exp \left[\left(-v \gamma+\frac{2 \sqrt{2 v \gamma}}{M}\right) \mathcal{O}\left(n^{3}\right)+\frac{2 \sqrt{2 v \gamma}}{M} \mathcal{O}\left(n^{2}\right)\right] \leq \exp \left(-n^{3} / M\right)
$$

when $M$ is sufficiently large. 
To complete the proof, we repeat the arguments that lead to the last assertion in Theorem 9. Because

$$
\left\{\theta \in \Theta: P T_{\theta}^{\gamma} \neq \varnothing\right\} \subseteq \bigcap \bigcup_{m \geq 1} \bigcup_{n \geq m} \mathcal{P}_{\text {cell center } x_{j}^{\left(4 n^{2}\right)}}^{x_{j}^{\left(4 n^{2}\right)}},
$$

and the probability of the RHS is no greater than

$$
\lim _{m \rightarrow \infty} \sum_{n \geq m} 2^{4 v n^{2}} e^{-n^{3} / M}=0,
$$

$P T_{\theta}^{\gamma}$ is the empty set with probability one.

We close this section with a remark on the universality of our main results with respect to different choices of regularization of the GFF.

Remark 14. The advantage of choosing the specific regularization $\bar{\theta}_{t}(x)$ is that it possesses technically desirable properties as discussed in Remark 4 and Lemma 5. However, the choice of regularization is not unique for the study of thick points. It is possible to consider a different family of "actions"

$$
\left\{\lambda_{t, x}: t \in(0,1], x \in \overline{S(O, 1)}\right\} \subseteq H^{-1}\left(\mathbb{R}^{v}\right)
$$

with $\lim _{t \searrow 0} \lambda_{t, x}=\delta_{x}$ in the sense of tempered distribution, use the corresponding Paley-Wiener integrals $\mathcal{I}\left(h_{\lambda_{t, x}}\right)(\theta)$ as the approximations of " $\theta(x)$ ", and define thick points accordingly. In this case the covariance function will be less explicit than (3.5), (3.7) and (3.8), but follows similar asymptotics as those established in Lemma 5. Thus, using the methods presented here (possibly with more technicality), one can obtain similar results on Hausdorff dimensions of thick point sets. However, whether two thick point sets (with respect to the same field instance) obtained via different regularization agree is a more complicated question. It is shown in [7] that, for log-correlated GFFs, thick point sets agree a.s. for regularization procedures that are "close" in a specific sense. It is worth investigating this issue for polynomial-correlated GFFs.

\section{Proof of the lower bound}

In this section we prove the lower bound (4.3). In fact, we will derive explicitly the Hausdorff dimension of a specific subset of $T_{\theta}^{\gamma}$, which leads to the desired lower bound of $\operatorname{dim}_{\mathcal{H}}\left(T_{\theta}^{\gamma}\right)$. The strategy is to study the convergence of $\frac{\bar{\theta}_{t}(x)}{\sqrt{-G(t) \ln t}}$ as $t \searrow 0$ along a prefixed sequence $\left\{r_{n}: n \geq 0\right\}$ that decays to zero sufficiently fast. The advantage of doing so is to reduce the problem, at least with respect to the parameter " $t$ ", from a continuum-parameter family to a discrete-parameter family. On the other hand, by making $r_{n}$ decay sufficiently fast, we are able to establish several important technical results on which the proof relies. We assume that $\left\{r_{n}: n \geq 0\right\}$ is a sequence of positive numbers satisfying that $r_{0}=1, r_{n} \searrow 0$ as $n \nearrow \infty$, and

$$
\lim _{n \rightarrow \infty} \frac{n \cdot \ln r_{n-1}}{\ln r_{n}}=0
$$

Definition 15. Let $\gamma \geq 0$. For each $\theta \in \Theta, x \in \overline{S(O, 1)}$ is called a sequential $\gamma$-thick point of $\theta$ with the sequence $\left\{r_{n}: n \geq 0\right\}$ if

$$
\lim _{n \rightarrow \infty} \frac{\bar{\theta}_{r_{n}}(x)}{\sqrt{-G\left(r_{n}\right) \ln r_{n}}}=\sqrt{2 v \gamma}
$$

With any sequence $\left\{r_{n}: n \geq 0\right\}$ as described above fixed, we denote by $S T_{\theta}^{\gamma}$ the collection of all the sequential $\gamma$-thick points of $\theta$ with $\left\{r_{n}: n \geq 0\right\}$. $S T_{\theta}^{\gamma}$ is a measurable subset of $\overline{S(O, 1)}$ and $S T_{\theta}^{\gamma} \subseteq T_{\theta}^{\gamma}$. The rest of this section will be devoted to determining $\operatorname{dim}_{\mathcal{H}}\left(S T_{\theta}^{\gamma}\right)$. In particular, we will prove the following result. 
Theorem 16. For $\gamma \in[0,1]$,

$$
\operatorname{dim}_{\mathcal{H}}\left(S T_{\theta}^{\gamma}\right)=v(1-\gamma) \quad \text { a.s. }
$$

Moreover, for $\mathcal{W}$-a.e. $\theta \in \Theta, x \in S T_{\theta}^{0}$ for Lebesgue-a.e. $x \in \overline{S(O, 1)}$. On the other hand, for $\gamma>1, S T_{\theta}^{\gamma}=\varnothing$ a.s.

Since $S T_{\theta}^{\gamma} \subseteq T_{\theta}^{\gamma}$, the established upper bounds of the size of $T_{\theta}^{\gamma}$ also apply to $S T_{\theta}^{\gamma}$, i.e., $\operatorname{dim}_{\mathcal{H}}\left(S T_{\theta}^{\gamma}\right) \leq v(1-\gamma)$ a.s. for $\gamma \in[0,1]$, and $S T_{\theta}^{\gamma}=\varnothing$ a.s. for $\gamma>1$. As for the lower bound, when $\gamma=0$, (3.6) implies that $\mathcal{W}\left(x \in S T_{\theta}^{0}\right)=1$ for every $x \in \overline{S(O, 1)}$. Let $\mu_{\text {Leb }}$ be the Lebesgue measure on $\mathbb{R}^{v}$, and $\mathcal{H}^{v}$ the $v$-dimensional Hausdorff measure on $\mathbb{R}^{v}$. Then, $\mathcal{H}^{v}=C_{v} \cdot \mu_{\text {Leb }}$ for some dimensional constant $C_{v}>0$. By Fubini's theorem,

$$
\mathbb{E}^{\mathcal{W}}\left[\mathcal{H}^{v}\left(S T_{\theta}^{0}\right)\right]=C_{v} \int_{\overline{S(O, 1)}} \mathcal{W}\left(x \in S T_{\theta}^{0}\right) \mu_{\mathrm{Leb}}(d x)=\mathcal{H}^{v}(\overline{S(O, 1)}) .
$$

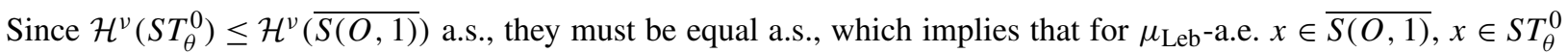
and hence $x \in T_{\theta}^{0}$. Thus, it is sufficient to derive the lower bound of $\operatorname{dim}_{\mathcal{H}}\left(S T_{\theta}^{\gamma}\right)$ for $\gamma \in(0,1)$.

Remark 17. One example of a sequence satisfying (5.1) is $\left\{r_{n}=2^{-2^{n^{2}}+1}: n \geq 0\right\}$. The method explained in this section applies to any sufficiently fast decaying sequence. However, we point out that the decay rate required by (5.1) is sufficient for our methods to work, but may not be necessary for the results in Theorem 16 to hold. On the other hand, for technical reasons, we will also assume that

$$
\ln \left(-\ln r_{n+1}\right)=o\left(-\ln r_{n}\right) \quad \text { for all large } n \text { 's. }
$$

This assumption will not reduce the generality of the method. If a given sequence $\left\{r_{n}: n \geq 0\right\}$ does not satisfy (5.2), one can always "fill in" more numbers to get a new sequence $\left\{\tilde{r}_{m}: m \geq 0\right\}$ that satisfies both (5.1) and (5.2), and the original sequence $\left\{r_{n}: n \geq 0\right\}$ is a subsequence of $\left\{\tilde{r}_{m}: m \geq 0\right\}$. Then, if we establish a lower bound of $\operatorname{dim}_{\mathcal{H}}\left(S T_{\theta}^{\gamma}\right)$ with $\left\{\tilde{r}_{m}: m \geq 0\right\}$, the lower bound also applies with any subsequence of $\left\{\tilde{r}_{m}: m \geq 0\right\}$.

The advantage of studying sequential thick points is that the same method can be applied to the study of other problems related to the geometry of GFFs, when convergence along sequence already gives rise to interesting objects. For example, recall that we mentioned in the Introduction about the Liouville quantum gravity (LQG) measures associated with log-correlated GFFs in 2D or higher dimensions. In fact, these random measures are constructed via a limiting procedure along a sequence of the regularized GFF (see, e.g., [3,5,15,21,22]), and consequently the supports of the LQG measures can be identified as the sets of sequential thick points corresponding to the same sequence. Although it remains open whether the analogs of the LQG measures for polynomial-correlated GFF exist, the study of sequential thick point sets, potentially as the supports of some random measures, is the natural first step in investigating this matter, especially in the absence of the perfect $\gamma$-thick point as pointed out in Theorem 13.

From now on, let $\gamma \in(0,1)$ and $\left\{r_{n}: n \geq 0\right\}$ satisfying (5.1) and (5.2) be fixed. To simplify the notation, for every $\theta \in \Theta, x \in \overline{S(O, 1)}$ and $n \geq 1$, we write

$$
\begin{aligned}
& \Delta \bar{\theta}_{n}(x):=\bar{\theta}_{r_{n}}(x)-\bar{\theta}_{r_{n-1}}(x), \\
& \Delta G_{n}:=G\left(r_{n}\right)-G\left(r_{n-1}\right) \text { and } \Delta D_{n}:=D\left(r_{n}\right)-D\left(r_{n-1}\right) .
\end{aligned}
$$

For each $x \in \overline{S(O, 1)}$, we define the following measurable subsets of $\Theta$ :

$$
P_{x, 0}:=\left\{\theta \in \Theta:\left|\bar{\theta}_{0}(x)-\sqrt{2 \nu \gamma} D\left(r_{0}\right)\right| \leq \sqrt{G\left(r_{0}\right)}\right\},
$$

and for $n \geq 1$,

$$
P_{x, n}:=\left\{\theta \in \Theta:\left|\Delta \bar{\theta}_{n}(x)-\sqrt{2 \nu \gamma} \Delta D_{n}\right| \leq \sqrt{\Delta G_{n}}\right\} \quad \text { and } \quad \Phi_{x, n}:=\left(\bigcap_{i=0}^{n} P_{x, i}\right) .
$$


The strategy we adopt here to obtain the lower bound of $\operatorname{dim}_{\mathcal{H}}\left(S T_{\theta}^{\gamma}\right)$ is similar to that appeared in [17], Section 3.2, where the counterpart result for log-correlated GFFs was established. The proof is carried out in multiple steps, with Step 1 being estimating the probabilities of $P_{x, n}$ and $\Phi_{x, n}$. Heuristically speaking, if $\theta \in \Phi_{x, n}$ as defined above, then the increment of $\bar{\theta}_{t}(x)$ over $\left[r_{i-1}, r_{i}\right]$, for each $i=1, \ldots, n$, follows a "desired" rate that is of the order of $\sqrt{2 v \gamma} \Delta D_{i}$, and hence summing over $i$ implies that $\bar{\theta}_{r_{n}}(x)$ is comparable with $\sqrt{2 v \gamma} D\left(r_{n}\right)$; in other words, if $\theta \in \Phi_{x, n}$, then $\bar{\theta}_{t}(x)$ is exhibiting behaviors, at least for $t$ up to $r_{n}$, that can potentially make $x$, as well as points that are sufficiently close to $x$, sequential $\gamma$-thick points of $\theta$. Based on this idea, in Step 2 we will identify a particular class of sequential $\gamma$-thick points. To be specific, for any fixed $\theta \in \Theta$, if $y \in \overline{S(O, 1)}$ satisfies that, for infinitely many $n$ 's, there exist $x^{(n)}$ with $\left|x^{(n)}-y\right|<r_{n}$ such that $\theta \in \Phi_{x^{(n)}, n}$, then $y$ must be a sequential $\gamma$-thick point of $\theta$, i.e., $y \in S T_{\theta}^{\gamma}$. Therefore, to bound $\operatorname{dim}_{\mathcal{H}}\left(S T_{\theta}^{\gamma}\right)$ from below, it suffices to study the lower bound of the Hausdorff dimension of the set consisting of such $y$ 's, and this will be carried out in Steps 3-5, via a standard application of Frostman's lemma.

Step 1: Derive the probability estimates.

Let $x \in \overline{S(O, 1)}$ be fixed. It is clear that $\left\{\bar{\theta}_{0}(x), \Delta \bar{\theta}_{n}(x), n \geq 1\right\}$ is a family of independent Gaussian random variables. The following simple facts about $P_{x, n}$ and $\Phi_{x, n}$ are in order.

Lemma 18. $P_{x, i}, i=0,1, \ldots, n$, are mutually independent. Moreover, for all sufficiently large $n$ 's,

$$
\mathcal{W}\left(P_{x, n}\right)=e^{\nu \gamma \ln r_{n}+\mathcal{O}\left(\sqrt{-\ln r_{n}}\right)}
$$

and

$$
\mathcal{W}\left(\Phi_{x, n}\right)=e^{\nu \gamma \ln r_{n}+o\left(-\ln r_{n}\right)} .
$$

The independence of $P_{x, i}$ 's simply follows from the observation in Remark 4. Since $\Delta G_{n}=\operatorname{Var}\left(\Delta \bar{\theta}_{n}(x)\right)$, the estimate (5.3) is a result of straightforward computations with Gaussian distributions. Finally, under the assumption (5.1) on $\left\{r_{n}: n \geq 0\right\}$, it is trivial to derive (5.4) from (5.3). Detailed proofs are omitted.

Step 2: Obtain a subset of $S T_{\theta}^{\gamma}$.

As mentioned above, in this step we will identify a specific class of sequential $\gamma$-thick points for every $\theta \in \Theta$. To this end, for every $n \geq 0$, we consider the lattice partition of $\overline{S(O, 1)}$ with cell size $r_{n}$, let $\mathcal{K}_{n}:=\left\{x_{j}^{(n)}: j=1, \ldots, K_{n}\right\}$ be the collection of all the cell centers where $K_{n}:=r_{n}^{-\nu}$, and for every $\theta \in \Theta$, set

$$
\Xi_{n, \theta}:=\left\{x_{j}^{(n)} \in \mathcal{K}_{n}: 1 \leq j \leq K_{n}, \theta \in \Phi_{x_{j}^{(n)}, n}\right\} .
$$

Then, we can prove the following result.

Lemma 19. For every $\theta \in \Theta$,

$$
S T_{\theta}^{\gamma} \supseteq \Sigma_{\theta}^{\gamma}:=\bigcap_{k \geq 1} \overline{\bigcup_{n \geq k} S\left(x, \Xi_{n, \theta}\right)}
$$

Proof. Let $\theta \in \Theta$ be fixed. We first show that

$$
S T_{\theta}^{\gamma} \supseteq \bigcap_{k \geq 1} \bigcup_{n \geq k} \bigcup_{x \in \Xi_{n, \theta}} \overline{S\left(x, r_{n}\right)}
$$

For any $y$ in the RHS above, there exists a subsequence $\left\{n_{k}: k \geq 1\right\} \subseteq \mathbb{N}$ with $n_{k} \nearrow \infty$ as $k \nearrow \infty$ and a sequence of cell centers $\left\{x^{\left(n_{k}\right)} \in \Xi_{n_{k}, \theta}: k \geq 1\right\}$ such that $\left|y-x^{\left(n_{k}\right)}\right| \leq \sqrt{\nu} r_{n_{k}}$ for every $k \geq 1$. Moreover, by the definition of $\Xi_{n_{k}, \theta}$ 
and the triangle inequality, for every $j=0,1, \ldots, n_{k}$,

$$
\left|\frac{\bar{\theta}_{r_{j}}\left(x^{\left(n_{k}\right)}\right)}{D\left(r_{j}\right)}-\sqrt{2 v \gamma}\right| \leq \frac{\sqrt{G\left(r_{0}\right)}+\sum_{p=1}^{j} \sqrt{\Delta G_{p}}}{D\left(r_{j}\right)} \leq \frac{j+1}{\sqrt{-\ln r_{j}}} .
$$

When $j$ is sufficiently large, the RHS above can be arbitrarily small, which can be easily derived from (5.1); moreover, (4.7) implies that, if $n_{k}$ is large such that $r_{n_{k}}<r_{j+1}^{4}$, then

$$
\left|\frac{\bar{\theta}_{r_{j}}\left(x^{\left(n_{k}\right)}\right)}{D\left(r_{j}\right)}-\frac{\bar{\theta}_{r_{j}}(y)}{D\left(r_{j}\right)}\right| \leq r_{j-1}^{3 / 16} .
$$

It follows immediately from the triangle inequality that

$$
\lim _{j \rightarrow \infty} \frac{\bar{\theta}_{r_{j}}(y)}{D\left(r_{j}\right)}=\sqrt{2 v \gamma},
$$

and hence $y \in S T_{\theta}^{\gamma}$.

Next, let $\tilde{y} \in \Sigma_{\theta}^{\gamma}$. For each $k \geq 1$, there exists a sequence $\left\{y_{p}: p \geq 1\right\}$ with

$$
y_{p} \in \bigcup_{n \geq k} \bigcup_{x \in \Xi_{n, \theta}} S\left(x, r_{n}\right) \quad \text { for every } p \geq 1
$$

such that $\lim _{p \rightarrow \infty} y_{p}=\tilde{y}$. Either, for some $n \geq k, y_{p} \in \bigcup_{x \in \Xi_{n, \theta}} S\left(x, r_{n}\right)$ for infinitely many $p$ 's, in which case there must exist $x^{(n)} \in \Xi_{n, \theta}$ such that $\left|\tilde{y}-x^{(n)}\right| \leq 2 \sqrt{v} \cdot r_{n}$, or, one can find a subsequence $\left\{n_{p}: p \geq 0\right\}$ with $n_{p} \nearrow \infty$ as $p \nearrow \infty$ such that $y_{p} \in S\left(x^{\left(n_{p}\right)}, r_{n_{p}}\right)$ for some $x^{\left(n_{p}\right)} \in \Xi_{n_{p}, \theta}$, in which case, since $y_{p} \rightarrow \tilde{y},\left|x^{\left(n_{p}\right)}-\tilde{y}\right|$ can be arbitrarily small when $p$ is sufficiently large. In either case, one can follow similar arguments as above to show that $\tilde{y} \in S T_{\theta}^{\gamma}$.

We now have obtained $\Sigma_{\theta}^{\gamma}$, a subset of $S T_{\theta}^{\gamma}$. The advantage of $\Sigma_{\theta}^{\gamma}$ is that it takes an explicit form in which only simple Euclidean sets (i.e., squares $S\left(x, r_{n}\right)$ ) are involved. Our goal is to apply Frostman's lemma to estimate $\operatorname{dim}_{\mathcal{H}}\left(\Sigma_{\theta}^{\gamma}\right)$ from below, which requires us to find a Borel measure $\mu_{\theta}$ supported on $\Sigma_{\theta}^{\gamma}$ and to study the $\alpha$-energy ${ }^{11}$ of $\mu_{\theta}$ for certain $\alpha>0$. We will obtain $\mu_{\theta}$ via a limiting process by first considering a naturally chosen family of Borel measures $\mu_{n, \theta}$ supported on $\overline{\bigcup_{x \in \Xi_{n, \theta}} S\left(x, r_{n}\right)}$ for $n \geq 1$. Below in Step 3 and Step 4, after formulating $\mu_{n, \theta}$ as random finite measures on $\overline{S(O, 1)}$, we will investigate the second moment (with respect to the Gaussian measure $\mathcal{W}$ ) of $\mu_{n, \theta}(\overline{S(O, 1)})$, as well as the $\alpha$-energy of $\mu_{n, \theta}$ over $\overline{S(O, 1)}$, aiming at bounding both quantities uniformly in $n \geq 1$. As one will see soon, the key ingredient in achieving both bounds is to control the following quotients:

$$
\frac{\mathcal{W}\left(\Phi_{x_{j}^{(n)}, n} \cap \Phi_{x_{k}^{(n)}, n}\right)}{\mathcal{W}\left(\Phi_{x_{j}^{(n)}, n}\right) \mathcal{W}\left(\Phi_{x_{k}^{(n)}, n}\right)} \quad \text { for } x_{j}^{(n)}, x_{k}^{(n)} \in \mathcal{K}_{n}
$$

The method that was used in [17] to treat the quotients above for log-correlated GFFs is not fully applicable in our setting. We have to carry out a more careful analysis of these quotients by separating the cases according to a finer decomposition of the range of $\left|x_{j}^{(n)}-x_{k}^{(n)}\right|$. As a result, the work in Step 3 and Step 4 is considerably heavy in technicality. For the sake of exposition, we leave some of the lengthy and technical proofs in the Appendix.

\section{Step 3: Construct a family of random measures.}

For each $n \geq 1$ and $\theta \in \Theta$, define a finite measure on $\overline{S(O, 1)}$ by,

$$
\forall A \in \mathcal{B}(\overline{S(O, 1)}), \quad \mu_{n, \theta}(A):=\frac{1}{K_{n}} \sum_{j=1}^{K_{n}} \frac{\mathbb{I}_{\Xi_{n, \theta}}\left(x_{j}^{(n)}\right)}{\mathcal{W}\left(\Phi_{x_{j}^{(n)}, n}\right)} \frac{\operatorname{vol}\left(A \cap S\left(x_{j}^{(n)}, r_{n}\right)\right)}{\operatorname{vol}\left(S\left(x_{j}^{(n)}, r_{n}\right)\right)},
$$

\footnotetext{
${ }^{11}$ The $\alpha$-energy of a finite Borel measure on $\overline{S(O, 1)}$ is defined in Step 4 (5.17).
} 
where "vol" refers to the volume under the Lebesgue measure on $\mathbb{R}^{v}$. It is clear that $\mu_{n, \theta}$ is a measure-valued random variable on $\Theta$, and

$$
\mathbb{E}^{\mathcal{W}}\left[\mu_{n, \theta}(\overline{S(O, 1)})\right]=1
$$

for every $n \geq 1$. We also need to study the second moment of $\mu_{n, \theta}(\overline{S(O, 1)})$, to which end we write the second moment as

$$
\mathbb{E}^{\mathcal{W}}\left[\left(\mu_{n, \theta}(\overline{S(O, 1)})\right)^{2}\right]=\frac{1}{K_{n}^{2}} \sum_{j, k=1}^{K_{n}} \frac{\mathcal{W}\left(\Phi_{x_{j}^{(n)}, n} \cap \Phi_{x_{k}^{(n)}, n}\right)}{\mathcal{W}\left(\Phi_{x_{j}^{(n)}, n}\right) \mathcal{W}\left(\Phi_{x_{k}^{(n)}, n}\right)}
$$

Our goal is to show that

$$
\sup _{n \geq 1} \mathbb{E}^{\mathcal{W}}\left[\left(\mu_{n, \theta}(\overline{S(O, 1)})\right)^{2}\right]<\infty .
$$

First notice that, when $j=k$, (5.4) implies that

$$
\frac{\mathcal{W}\left(\Phi_{x_{j}^{(n)}, n} \cap \Phi_{x_{k}^{(n)}, n}\right)}{\mathcal{W}\left(\Phi_{x_{j}^{(n)}, n}\right) \mathcal{W}\left(\Phi_{x_{k}^{(n)}, n}\right)}=\frac{1}{\mathcal{W}\left(\Phi_{x_{j}^{(n)}, n}\right)} \leq e^{-\nu \gamma \ln r_{n}+o\left(-\ln r_{n}\right)}
$$

so the sum over the diagonal terms in (5.8) is bounded from above by

$$
K_{n}^{-1} \cdot e^{-\nu \gamma \ln r_{n}+o\left(-\ln r_{n}\right)}=e^{(\nu-\nu \gamma) \ln r_{n}+o\left(-\ln r_{n}\right)},
$$

which converges to zero as $n \rightarrow \infty$ so long as $\gamma<1$. So we only need to treat the sum over the off-diagonal terms in (5.8), and this is done in separate cases depending on the distance between the two cell centers $x_{j}^{(n)}$ and $x_{k}^{(n)}$.

Assume that $j \neq k$. Then there exists a unique $i \in \mathbb{N}, 0 \leq i \leq n-1$, such that

$$
2 r_{i+1} \leq\left|x_{j}^{(n)}-x_{k}^{(n)}\right|<2 r_{i},
$$

we can rewrite the sum over the off-diagonal terms in (5.8) as

$$
\frac{1}{K_{n}^{2}} \sum_{j=1}^{K_{n}} \sum_{i=0}^{n-1} \sum_{\{k:(\dagger)} \frac{\mathcal{W}\left(\Phi_{x_{j}^{(n)}, n} \cap \Phi_{x_{k}^{(n)}, n}\right)}{\mathcal{W}\left(\Phi_{x_{j}^{(n)}, n}\right) \mathcal{W}\left(\Phi_{x_{k}^{(n)}, n}\right)}
$$

Let $j$ and $k$ be fixed for now. For $l, l^{\prime} \geq 1$, set

$$
\operatorname{DCov}\left(l, l^{\prime}\right):=\mathbb{E}^{\mathcal{W}}\left[\Delta \bar{\theta}_{l}\left(x_{j}^{(n)}\right) \cdot \Delta \bar{\theta}_{l^{\prime}}\left(x_{k}^{(n)}\right)\right] .
$$

By (3.2), $\operatorname{DCov}\left(l, l^{\prime}\right)$ only depends on $r_{l}, r_{l^{\prime}}$ and $\left|x_{j}^{(n)}-x_{k}^{(n)}\right|$. It is sufficient to treat the cases when $\left|x_{j}^{(n)}-x_{k}^{(n)}\right|$ is small, or equivalently, when $i$, as determined by $(\dagger)$, is large. One can easily use (3.7) and (3.8) to verify that $\operatorname{DCov}\left(l, l^{\prime}\right)=0$ when $l^{\prime} \geq i+2$ and either $l \geq i+2$ or $l \leq i-1$, which implies that the family

$$
\left\{\Delta \bar{\theta}_{l}\left(x_{j}^{(n)}\right), \Delta \bar{\theta}_{l^{\prime}}\left(x_{k}^{(n)}\right): 1 \leq l \leq i-1, i+2 \leq l \leq n, i+2 \leq l^{\prime} \leq n\right\}
$$

is independent. However, the independence of this family alone is not sufficient for (5.9) to be bounded in $n$, mainly because $r_{i+1} \ll r_{i}$ which means that the range of $\left|x_{j}^{(n)}-x_{k}^{(n)}\right|$ given by $(\dagger)$ is too "coarse". To proceed, we need to further break down the range of $\left|x_{j}^{(n)}-x_{k}^{(n)}\right|$ in such a way that we can treat each case separately by fully exploiting the covariance properties (3.7) and (3.8) of the family of $\bar{\theta}_{t}(x)$. The figure below (Figure 1) shows how we will further divide the interval $\left[2 r_{i+1}, 2 r_{i}\right.$ ) into multiple sections. As marked in the figure, Case 1 and Case 2 correspond to relatively "narrow" sections, and the small widths of the intervals will help us easily control the RHS of (5.9). For all the other cases, the key is to treat the probability of the intersection of $\left(P_{x_{j}^{(n)}, l}\right)$ 's and $\left(P_{x_{k}^{(n)}, l^{\prime}}\right)$ 's for $l$ and $l^{\prime}$ close to the cut-off of each section. 


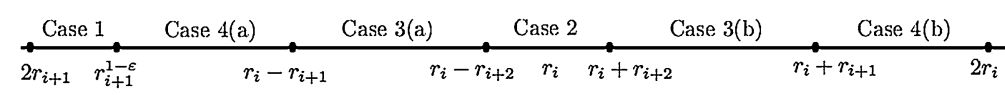

Fig. 1. Separate cases according to the range of $\left|x_{j}^{(n)}-x_{k}^{(n)}\right|$.

\section{Case 1.}

Assume that, for some sufficiently small $\epsilon \in(0,1-\gamma)$,

$$
2 r_{i+1} \leq\left|x_{j}^{(n)}-x_{k}^{(n)}\right|<r_{i+1}^{1-\varepsilon} .
$$

In this case, besides the family of independent random variables in (5.10), we also have that for $l^{\prime} \geq i+2$,

$$
B\left(x_{k}^{(n)}, r_{l^{\prime}-1}\right) \subseteq B\left(x_{j}^{(n)}, r_{i}\right) \quad \text { and } \quad B\left(x_{j}^{(n)}, r_{i+1}\right) \cap B\left(x_{k}^{(n)}, r_{l^{\prime}-1}\right)=\varnothing
$$

which, by (3.7) and (3.8), leads to $\operatorname{DCov}\left(i ; l^{\prime}\right)=0$ and $\operatorname{DCov}\left(i+1, l^{\prime}\right)=0$, and hence $\Delta \bar{\theta}_{i}\left(x_{j}^{(n)}\right)$ and $\Delta \bar{\theta}_{i+1}\left(x_{j}^{(n)}\right)$ are independent of $\Delta \bar{\theta}_{l^{\prime}}\left(x_{k}^{(n)}\right)$. As a result,

$$
\begin{aligned}
\frac{\mathcal{W}\left(\Phi_{x_{j}^{(n)}, n} \cap \Phi_{x_{k}^{(n)}, n}\right)}{\mathcal{W}\left(\Phi_{x_{j}^{(n)}, n}\right) \mathcal{W}\left(\Phi_{x_{k}^{(n)}, n}\right)} & \leq \frac{\mathcal{W}\left(\Phi_{x_{j}^{(n)}, n}\right) \cdot \prod_{l^{\prime}=i+2}^{n} \mathcal{W}\left(P_{x_{k}^{(n)}, l^{\prime}}\right)}{\mathcal{W}\left(\Phi_{x_{j}^{(n)}, n}\right) \mathcal{W}\left(\Phi_{x_{k}^{(n)}, n}\right)} \\
& =\frac{1}{\mathcal{W}\left(\Phi_{x_{k}^{(n)}, i+1}\right)} \leq \exp \left[-v \gamma \ln r_{i+1}+o\left(-\ln r_{i+1}\right)\right] .
\end{aligned}
$$

The last inequality follows from (5.4). On the other hand, if $j$ is fixed, then the number of $x_{k}^{(n)}$,s such that

$$
2 r_{i+1} \leq\left|x_{j}^{(n)}-x_{k}^{(n)}\right|<r_{i+1}^{1-\varepsilon}
$$

is of the order of $\left(r_{i+1}^{1-\epsilon} / r_{n}\right)^{\nu}$. The contribution to (5.9) under this case is

$$
\sum_{i=0}^{n-1} \exp \left[v(1-\epsilon-\gamma) \ln r_{i+1}+o\left(-\ln r_{i+1}\right)\right]
$$

which is bounded in $n$ since $\epsilon<1-\gamma$.

Case 2.

Assume that

$$
r_{i}-r_{i+2}<\left|x_{j}^{(n)}-x_{k}^{(n)}\right| \leq r_{i}+r_{i+2} .
$$

Since the random variables in (5.10) are independent, we have that

$$
\begin{aligned}
\frac{\mathcal{W}\left(\Phi_{x_{j}^{(n)}, n} \cap \Phi_{x_{k}^{(n)}, n}\right)}{\mathcal{W}\left(\Phi_{x_{j}^{(n)}, n}\right) \mathcal{W}\left(\Phi_{x_{k}^{(n)}, n}\right)} & \leq \frac{\mathcal{W}\left(\Phi_{x_{j}^{(n)}, i-1}\right) \prod_{l=i+2}^{n} \mathcal{W}\left(P_{x_{j}^{(n)}, l}\right) \prod_{l^{\prime}=i+2}^{n} \mathcal{W}\left(P_{x_{k}^{(n)}, l^{\prime}}\right)}{\mathcal{W}\left(\Phi_{x_{j}^{(n)}, n}\right) \mathcal{W}\left(\Phi_{x_{k}^{(n)}, n}\right)} \\
& =\frac{1}{\mathcal{W}\left(P_{x_{j}^{(n)}, i}\right) \mathcal{W}\left(P_{x_{j}^{(n)}, i+1}\right) \mathcal{W}\left(\Phi_{x_{k}^{(n)}, i+1}\right)},
\end{aligned}
$$

which, by (5.3) and (5.4), is no greater than

$$
\exp \left[-3 v \gamma \ln r_{i+1}+o\left(-\ln r_{i+1}\right)\right]
$$


Meanwhile, with $x_{j}^{(n)}$ fixed, the number of $x_{k}^{(n)}$ 's that satisfy (5.11) is of the order of $r_{i}^{\nu-1} r_{i+2} / r_{n}^{\nu}$. Hence, the contribution to (5.9) under this case is

$$
\sum_{i=0}^{n-1} \exp \left[\ln r_{i+2}-3 v \gamma \ln r_{i+1}+o\left(-\ln r_{i+1}\right)\right]
$$

which is bounded in $n$ by the assumption (5.1).

Case 3

Assume that either

$$
r_{i}-r_{i+1}<\left|x_{j}^{(n)}-x_{k}^{(n)}\right| \leq r_{i}-r_{i+2}
$$

or

$$
r_{i}+r_{i+2} \leq\left|x_{j}^{(n)}-x_{k}^{(n)}\right|<r_{i}+r_{i+1}
$$

We observe that for all $l^{\prime} \geq i+3$, by (3.7) and (3.8), under the hypothesis (3a) or (3b), DCov $\left(i+1, l^{\prime}\right)=0$. Together with the family of independent random variables in (5.10), we see that both $P_{x_{j}^{(n)}, i+1}$ and $P_{x_{j}^{(n)}, i+2}$ are independent of $P_{x_{k}^{(n)}, l^{\prime}}$ for all $l^{\prime} \geq i+3$, and similarly both $P_{x_{k}^{(n)}, i+1}$ and $P_{x_{k}^{(n)}, i+2}$ are independent of $P_{x_{j}^{(n)}, l}$ for all $l \geq i+3$. Thus, $\mathcal{W}\left(\Phi_{x_{j}^{(n)}, n} \cap \Phi_{x_{k}^{(n)}, n}\right)$ is bounded from above by

$$
\prod_{l=i+3}^{n} \mathcal{W}\left(P_{x_{j}^{(n)}, l}\right) \cdot \prod_{l^{\prime}=i+3}^{n} \mathcal{W}\left(P_{x_{k}^{(n)}, l^{\prime}}\right) \mathcal{W}\left(P_{x_{j}^{(n)}, i+1} \cap P_{x_{j}^{(n)}, i+2} \cap P_{x_{k}^{(n)}, i+1} \cap P_{x_{k}^{(n)}, i+2}\right),
$$

so we only need to focus on the family

$$
\left\{\Delta \bar{\theta}_{i+1}\left(x_{j}^{(n)}\right), \Delta \bar{\theta}_{i+2}\left(x_{j}^{(n)}\right), \Delta \bar{\theta}_{i+1}\left(x_{k}^{(n)}\right), \Delta \bar{\theta}_{i+2}\left(x_{k}^{(n)}\right)\right\} .
$$

After a lengthy computation through multiple stages of conditioning, we can derive the estimate below. Here we only state the result and leave the proof in the Appendix.

Lemma 20. Under the hypothesis (3a) or (3b), there exists a constant $C_{v}>0$ that is universal in $i \geq 0$ such that

$$
\frac{\mathcal{W}\left(P_{x_{j}^{(n)}, i+1} \cap P_{x_{j}^{(n)}, i+2} \cap P_{x_{k}^{(n)}, i+1} \cap P_{x_{k}^{(n)}, i+2}\right)}{\mathcal{W}\left(P_{x_{j}^{(n)}, i+1}\right) \mathcal{W}\left(P_{x_{j}^{(n)}, i+2}\right) \mathcal{W}\left(P_{x_{k}^{(n)}, i+1}\right) \mathcal{W}\left(P_{x_{k}^{(n)}, i+2}\right)} \leq e^{C_{\nu} \sqrt{-\ln r_{i+1}}} .
$$

Then, it follows from (5.1), (5.12) and (5.13) that, in Case 3,

$$
\begin{aligned}
\frac{\mathcal{W}\left(\Phi_{x_{j}^{(n)}, n} \cap \Phi_{x_{k}^{(n)}, n}\right)}{\mathcal{W}\left(\Phi_{x_{j}^{(n)}, n}\right) \mathcal{W}\left(\Phi_{x_{k}^{(n)}, n}\right)} & \leq \frac{\mathcal{W}\left(P_{x_{j}^{(n)}, i+1} \cap P_{x_{j}^{(n)}, i+2} \cap P_{x_{k}^{(n)}, i+1} \cap P_{x_{k}^{(n)}, i+2}\right)}{\mathcal{W}\left(\Phi_{x_{j}^{(n)}, i+2}\right) \mathcal{W}\left(\Phi_{x_{k}^{(n)}, i+2}\right)} \\
& \leq \frac{\exp \left(C_{v} \sqrt{-\ln r_{i+1}}\right)}{\mathcal{W}\left(\Phi_{x_{j}^{(n)}, i}\right) \mathcal{W}\left(\Phi_{x_{k}^{(n)}, i}\right)}=\exp \left[o\left(-\ln r_{i+1}\right)\right] .
\end{aligned}
$$

On the other hand, with $x_{j}^{(n)}$ fixed, the number of $x_{k}^{(n)}$, s that satisfy either (3a) or (3b) is of the order of $r_{i}^{\nu-1} r_{i+1} / r_{n}^{\nu}$. Hence, the contribution to (5.9) under this case is

$$
\sum_{i=0}^{n-1} \exp \left[\ln r_{i+1}+o\left(-\ln r_{i+1}\right)\right]
$$

which is bounded in $n$. 
Case 4.

The last case is that either

$$
r_{i+1}^{1-\epsilon}<\left|x_{j}^{(n)}-x_{k}^{(n)}\right| \leq r_{i}-r_{i+1},
$$

or

$$
r_{i}+r_{i+1} \leq\left|x_{j}^{(n)}-x_{k}^{(n)}\right|<2 r_{i},
$$

The strategy for studying this case is similar to that for the previous case. We will omit the technical details that are the same as earlier, but only address the differences in the treatment of Case 4 from that of Case 3 . When (4a) or (4b) applies, one can use (3.7) and (3.8) to verify that both $P_{x_{j}^{(n)}, i}$ and $P_{x_{j}^{(n)}, i+1}$ are independent of $P_{x_{k}^{(n)}, l^{\prime}}$ for all $l^{\prime} \geq i+2$, and $P_{x_{k}^{(n)}, i+1}$ is independent of $P_{x_{j}^{(n)}, l}$ for all $l \geq i+2$. Thus, $\mathcal{W}\left(\Phi_{x_{j}^{(n)}, n} \cap \Phi_{x_{k}^{(n)}, n}\right)$ is no greater than

$$
\mathcal{W}\left(\bigcap_{l=i+2}^{n} P_{x_{j}^{(n)}, l}\right) \mathcal{W}\left(\bigcap_{l^{\prime}=i+2}^{n} P_{x_{k}^{(n)}, l^{\prime}}\right) \cdot \mathcal{W}\left(P_{x_{j}^{(n)}, i} \cap P_{x_{j}^{(n)}, i+1} \cap P_{x_{k}^{(n)}, i+1}\right) .
$$

Again, we state the key probability estimate below and leave the proof in the Appendix.

Lemma 21. Under the hypothesis (4a) or (4b), there exists a constant $C_{v, \epsilon}>0$ that is universal in $i \geq 0$ such that

$$
\frac{\mathcal{W}\left(P_{x_{j}^{(n)}, i} \cap P_{x_{j}^{(n)}, i+1} \cap P_{x_{k}^{(n)}, i+1}\right)}{\mathcal{W}\left(P_{x_{j}^{(n)}, i}\right) \mathcal{W}\left(P_{x_{j}^{(n)}, i+1}\right) \mathcal{W}\left(P_{x_{k}^{(n)}, i+1}\right)} \leq \exp \left(C_{v, \epsilon} \sqrt{-\ln r_{i}}\right) .
$$

Based on (5.4), (5.14) and (5.15), we see that, in Case 4,

$$
\begin{aligned}
\frac{\mathcal{W}\left(\Phi_{x_{j}^{(n)}, n} \cap \Phi_{x_{k}^{(n)}, n}\right)}{\mathcal{W}\left(\Phi_{x_{j}^{(n)}, n}\right) \mathcal{W}\left(\Phi_{x_{k}^{(n)}, n}\right)} & \leq \frac{\mathcal{W}\left(P_{x_{j}^{(n)}, i} \cap P_{x_{j}^{(n)}, i+1} \cap P_{x_{k}^{(n)}, i+1}\right)}{\mathcal{W}\left(\Phi_{x_{j}^{(n)}, i+1}\right) \mathcal{W}\left(\Phi_{x_{k}^{(n)}, i+1}\right)} \\
& \leq \frac{\exp \left(C_{\nu, \epsilon} \sqrt{-\ln r_{i}}\right)}{\mathcal{W}\left(\Phi_{x_{j}^{(n)}, i-1}\right) \mathcal{W}\left(\Phi_{x_{k}^{(n)}, i}\right)} \\
& \leq \exp \left[-v \gamma \ln r_{i}+o\left(-\ln r_{i}\right)\right]
\end{aligned}
$$

With $x_{j}^{(n)}$ is fixed, the number of $x_{k}^{(n)}$, s that satisfy either (4a) or (4b) is of the order of $\left(2 r_{i}\right)^{v} / r_{n}^{v}$. Hence, the contribution to (5.9) under this case is

$$
\sum_{i=0}^{n-1} \exp \left[v(1-\gamma) \ln r_{i}+o\left(-\ln r_{i}\right)\right]
$$

which is bounded in $n$ since $\gamma<1$.

Summarizing our findings in all the cases above, we conclude that

$$
\sup _{n \geq 1} \mathbb{E}^{\mathcal{W}}\left[\left(\mu_{n, \theta}(\overline{S(O, 1)})\right)^{2}\right]<\infty
$$

Step 4: Study the $\alpha$-energy of $\mu_{n, \theta}$.

Besides bounding the second moment of $\mu_{n, \theta}(\overline{S(O, 1)})$, another ingredient we need is the boundedness of the expected $\alpha$-energy of $\mu_{n, \theta}$ for certain $\alpha>0$. Namely, for fixed $\alpha>0, \theta \in \Theta$ and $n \geq 1$, we will consider the $\alpha$-energy of $\mu_{n, \theta}$ 
over $\overline{S(O, 1)}$ which is given by

$$
I_{\alpha}\left(\mu_{n, \theta}\right):=\int_{\overline{S(O, 1)}} \int_{\overline{S(O, 1)}}|y-w|^{-\alpha} \mu_{n, \theta}(d y) \mu_{n, \theta}(d w) .
$$

By the definition of $\mu_{n, \theta}(5.6), \mathbb{E}^{\mathcal{W}}\left[I_{\alpha}\left(\mu_{n, \theta}\right)\right]$ is equal to

$$
\frac{1}{K_{n}^{2}} \sum_{j, k=1}^{K_{n}} \frac{\mathcal{W}\left(\Phi_{x_{j}^{(n)}, n} \cap \Phi_{x_{k}^{(n)}, n}\right)}{\mathcal{W}\left(\Phi_{x_{j}^{(n)}, n}\right) \mathcal{W}\left(\Phi_{x_{k}^{(n)}, n}\right)} \frac{\int \frac{}{S\left(x_{j}^{(n)}, r_{n}\right)} \int \frac{}{S\left(x_{k}^{(n)}, r_{n}\right)}|y-w|^{-\alpha} d y d w}{\operatorname{vol}\left(S\left(x_{j}^{(n)}, r_{n}\right)\right) \operatorname{vol}\left(S\left(x_{k}^{(n)}, r_{n}\right)\right)}
$$

In this subsection we will show that, if $0<\alpha<v(1-\gamma)$, then

$$
\sup _{n \geq 1} \mathbb{E}^{\mathcal{W}}\left[I_{\alpha}\left(\mu_{n, \theta}\right)\right]<\infty
$$

The arguments we use here are slight variations of those in Step 3. For simplicity, we write

$$
I\left(x_{j}^{(n)}, x_{k}^{(n)}\right):=\frac{\int \frac{}{S\left(x_{j}^{(n)}, r_{n}\right)} \int \frac{}{S\left(x_{k}^{(n)}, r_{n}\right)}|y-w|^{-\alpha} d y d w}{\operatorname{vol}\left(S\left(x_{j}^{(n)}, r_{n}\right)\right) \operatorname{vol}\left(S\left(x_{k}^{(n)}, r_{n}\right)\right)} .
$$

When $j=k$, so long as $\alpha<v, I\left(x_{j}^{(n)}, x_{k}^{(n)}\right)=C_{v} \cdot r_{n}^{-\alpha}$ for some constant $C_{v}>0$. Therefore, the sum over the terms along the diagonal in (5.18) is

$$
\frac{1}{K_{n}^{2}} \sum_{j=1}^{K_{n}} \frac{C_{v} \cdot r_{n}^{-\alpha}}{\mathcal{W}\left(\Phi_{x_{j}^{(n)}, n}\right)} \leq C_{v} \cdot \exp \left\{[v(1-\gamma)-\alpha] \ln r_{n}+o\left(-\ln r_{n}\right)\right\}
$$

which tends to zero as $n \rightarrow \infty$ whenever $\alpha<v(1-\gamma)$. So it is sufficient to treat the sum over the off-diagonal terms in (5.18). To this end, we follow a similar approach as the one adopted in the previous step. Again, assume that $j \neq k$, let $i \in \mathbb{N}, 0 \leq i \leq n-1$, be the unique integer such that

$$
2 r_{i+1} \leq\left|x_{j}^{(n)}-x_{k}^{(n)}\right|<2 r_{i}
$$

and we rewrite the sum over the off-diagonal terms in (5.18) as

$$
\frac{1}{K_{n}^{2}} \sum_{j=1}^{K_{n}} \sum_{i=0}^{n-1} \sum_{\{k:(\dagger)} \frac{\mathcal{W}\left(\Phi_{x_{j}^{(n)}, n} \cap \Phi_{x_{k}^{(n)}, n}\right)}{\mathcal{W}\left(\Phi_{x_{j}^{(n)}, n}\right) \mathcal{W}\left(\Phi_{x_{k}^{(n)}, n}\right)} \cdot I\left(x_{j}^{(n)}, x_{k}^{(n)}\right) .
$$

Let $\alpha \in(0, v(1-\gamma))$ be fixed. We study the sum in (5.19) according to the four cases presented in the previous step. Same as earlier, it is sufficient to focus on the cases when $i$ is large.

Case 1 . Assume that for some $\epsilon \in\left(0,1-\gamma-\frac{\alpha}{v}\right)$,

$$
2 r_{i+1} \leq\left|x_{j}^{(n)}-x_{k}^{(n)}\right|<r_{i+1}^{1-\varepsilon} .
$$

We have found out in Case 1 previously (following the same arguments with a possibly smaller $\epsilon$ ) that

$$
\frac{\mathcal{W}\left(\Phi_{x_{j}^{(n)}, n} \cap \Phi_{x_{k}^{(n)}, n}\right)}{\mathcal{W}\left(\Phi_{x_{j}^{(n)}, n}\right) \mathcal{W}\left(\Phi_{x_{k}^{(n)}, n}\right)} \leq \exp \left[-v \gamma \ln r_{i+1}+o\left(-\ln r_{i+1}\right)\right]
$$


and with $x_{j}^{(n)}$ fixed, the number of $x_{k}^{(n)}$ 's that satisfy the criterion of Case 1 is of the order of $\left(r_{i+1}^{1-\epsilon} / r_{n}\right)^{\nu}$. Besides, it is easy to see that there exists $C_{v}>0$ such that $I\left(x_{j}^{(n)}, x_{k}^{(n)}\right) \leq C_{v} \cdot r_{i+1}^{-\alpha}$. So the contribution to (5.19) under this case is

$$
\sum_{i=0}^{n-1} \exp \left\{[v(1-\epsilon-\gamma)-\alpha] \ln r_{i+1}+o\left(-\ln r_{i+1}\right)\right\},
$$

which is bounded in $n$ since $\epsilon<1-\gamma-\frac{\alpha}{v}$.

Case 2, Case 3 and Case (4b). Under any of the conditions, as imposed in the previous step, of these three cases, we have that $I\left(x_{j}^{(n)}, x_{k}^{(n)}\right) \leq C_{v} \cdot r_{i}^{-\alpha}$. Combining this with the findings from the previous step, i.e., the estimate on

$$
\frac{\mathcal{W}\left(\Phi_{x_{j}^{(n)}, n} \cap \Phi_{x_{k}^{(n)}, n}\right)}{\mathcal{W}\left(\Phi_{x_{j}^{(n)}, n}\right) \mathcal{W}\left(\Phi_{x_{k}^{(n)}, n}\right)}
$$

and the number of qualifying $k$ 's for any fixed $j$, one can easily confirm that the contribution to (5.19) under Case 2 or Case 3 or Case (4b), is bounded in $n$.

Case (4a). However, in the case of (4a), the arguments above will not work, since $r_{i}^{-\alpha}$ above would be replaced by $r_{i+1}^{-\alpha(1-\epsilon)}$. We need to apply a finer treatment by further decomposing the interval $\left(r_{i+1}^{1-\epsilon}, r_{i}-r_{i+1}\right]$ into a union of disjoint intervals. To be specific, let $Z$ be the smallest integer such that

$$
\left(r_{i+1} / r_{i}\right)^{(1-\gamma)^{Z}} \geq 1-\frac{r_{i+1}}{r_{i}},
$$

for which to happen it is sufficient to make

$$
(1-\gamma)^{Z} \leq \frac{\ln \left(1-r_{i+1} / r_{i}\right)}{\ln \left(r_{i+1} / r_{i}\right)},
$$

so $Z$ should be taken as approximately

$$
\frac{1}{\ln (1-\gamma)} \ln \left[\frac{\ln \left(1-r_{i+1} / r_{i}\right)}{\ln \left(r_{i+1} / r_{i}\right)}\right]+1=\mathcal{O}\left(-\ln r_{i+1}\right) .
$$

Define a sequence of positive numbers $\left\{R_{m}: m=0, \ldots, Z\right\}$ by $R_{0}:=r_{i+1}^{1-\epsilon}$, and

$$
R_{m}:=r_{i+1}^{(1-\gamma)^{m}} \cdot r_{i}^{1-(1-\gamma)^{m}} \text { for } m=1, \ldots, Z .
$$

Clearly, $R_{m}<R_{m+1}$ and

$$
R_{Z}=r_{i} \cdot\left(r_{i+1} / r_{i}\right)^{(1-\gamma)^{Z}} \geq r_{i}-r_{i+1} .
$$

Denote $U_{m}:=\left(R_{m}, R_{m+1}\right]$ for $m=0,1, \ldots, Z-1$. Clearly,

$$
\left(r_{i+1}^{1-\epsilon}, r_{i}-r_{i+1}\right] \subseteq \bigcup_{m=0}^{Z-1} U_{m}
$$

For each $m=0,1, \ldots, Z$, if $\left|x_{j}^{(n)}-x_{k}^{(n)}\right| \in U_{m}$, then $I\left(x_{j}^{(n)}, x_{k}^{(n)}\right) \leq C_{v} R_{m}^{-\alpha}$. Recall that, in Case 4,

$$
\frac{\mathcal{W}\left(\Phi_{x_{j}^{(n)}, n} \cap \Phi_{x_{k}^{(n)}, n}\right)}{\mathcal{W}\left(\Phi_{x_{j}^{(n)}, n}\right) \mathcal{W}\left(\Phi_{x_{k}^{(n)}, n}\right)} \leq \exp \left[-v \gamma \ln r_{i}+o\left(-\ln r_{i}\right)\right]
$$


Meanwhile, when $x_{j}^{(n)}$ is fixed, the number of $x_{k}^{(n)}$, s such that $\left|x_{j}^{(n)}-x_{k}^{(n)}\right| \in U_{m}$ is no greater than $R_{m+1}^{v} / r_{n}^{v}$. We will need the following estimate:

$$
\begin{aligned}
\exp & {\left[-v \gamma \ln r_{i}+o\left(-\ln r_{i}\right)\right] \cdot R_{m}^{-\alpha} \cdot R_{m+1}^{v} } \\
\leq & \exp \left\{\left[-v \gamma-\alpha+\alpha(1-\gamma)^{m}+v-v(1-\gamma)^{m+1}\right] \ln r_{i}+o\left(-\ln r_{i}\right)\right\} \\
& \quad \times \exp \left\{\left[-\alpha(1-\gamma)^{m}+v(1-\gamma)^{m+1}\right] \ln r_{i+1}\right\} \\
= & \exp \left\{[v(1-\gamma)-\alpha] \ln r_{i}+o\left(-\ln r_{i}\right)+[v(1-\gamma)-\alpha](1-\gamma)^{m} \ln \left(r_{i+1} / r_{i}\right)\right\} \\
\leq & \exp \left\{[v(1-\gamma)-\alpha] \ln r_{i}+o\left(-\ln r_{i}\right)\right\} .
\end{aligned}
$$

Hence, under the condition (4a), the contribution to (5.19) is

$$
\begin{aligned}
& \sum_{i=0}^{n-1} \sum_{m=0}^{Z-1} \exp \left[-v \gamma \ln r_{i}+o\left(-\ln r_{i}\right)\right] R_{m}^{-\alpha} \cdot R_{m+1}^{v} \\
& \quad \leq \sum_{i=0}^{n-1} Z \cdot \exp \left\{[v(1-\gamma)-\alpha] \ln r_{i}+o\left(-\ln r_{i}\right)\right\} \\
& \quad \leq \sum_{i=0}^{n-1} \exp \left\{[v(1-\gamma)-\alpha] \ln r_{i}+o\left(-\ln r_{i}\right)+\mathcal{O}\left(\ln \left(-\ln r_{i+1}\right)\right)\right\} \\
& \quad=\sum_{i=0}^{n-1} \exp \left\{[v(1-\gamma)-\alpha] \ln r_{i}+o\left(-\ln r_{i}\right)\right\},
\end{aligned}
$$

which is bounded in $n$ since $\alpha<v(1-\gamma)$. The last line is due to the second assumption (5.2) on the choice of $\left\{r_{n}: n \geq 0\right\}$.

Therefore, the work we carried out in Step 4 has led to the conclusion that for every $\alpha \in(0, v(1-\gamma))$,

$$
\sup _{n \geq 1} \mathbb{E}^{\mathcal{W}}\left[I_{\alpha}\left(\mu_{n, \theta}\right)\right]<\infty
$$

Step 5: Establish the lower bound of $\operatorname{dim}_{\mathcal{H}}\left(S T_{\theta}^{\gamma}\right)$.

To complete the proof of Theorem 16, we follow the same line of arguments as in [17] in the final step. Choose and fix any $\alpha \in(0, v(1-\gamma))$. After carefully treating the family of measures $\left\{\mu_{n, \theta}: n \geq 1\right\}$ in the previous two steps, we are now ready to apply a compactness argument to extract a limit measure $\mu_{\theta}$ that possesses desirable properties which will enable us to invoke Frostman's lemma. In particular, our goal is to show that for $\mathcal{W}$-a.e. $\theta \in \Theta$, $\mu_{\theta}$ assigns positive mass to $\Sigma_{\theta}^{\gamma}$, the subset of $S T_{\theta}^{\gamma}$ as defined in (5.5), and $I_{\alpha}\left(\mu_{\theta}\right)<\infty$. To achieve this goal, the results (5.16) and (5.20) obtained in the previous steps are essential. Set

$$
A_{1}:=\sup _{n \geq 1} \mathbb{E}^{\mathcal{W}}\left[\left(\mu_{n, \theta}(\overline{S(O, 1)})\right)^{2}\right] \quad \text { and } \quad A_{2}:=\sup _{n \geq 1} \mathbb{E}^{\mathcal{W}}\left[I_{\alpha}\left(\mu_{n, \theta}\right)\right] .
$$

For constants $c_{1}>1$ and $c_{2}>0$ which will be determined later, consider the measurable subsets of $\Theta$

$$
\Lambda_{n}^{\alpha:}:=\left\{\theta \in \Theta: \frac{1}{c_{1}} \leq \mu_{n, \theta}(\overline{S(O, 1)}) \leq c_{1}, I_{\alpha}^{\theta}\left(\mu_{n, \theta}\right) \leq c_{2}\right\}
$$

for $n \geq 1$, and set $\Lambda^{\alpha}:=\limsup _{n \rightarrow \infty} \Lambda_{n}^{\alpha}$. Clearly, simple applications of Markov's inequality lead to

$$
\sup _{n \geq 1} \mathcal{W}\left(I_{\alpha}^{\theta}\left(\mu_{n, \theta}\right)>c_{2}\right) \leq \frac{A_{2}}{c_{2}} \quad \text { and } \quad \sup _{n \geq 1} \mathcal{W}\left(\mu_{n, \theta}(\overline{S(O, 1)})>c_{1}\right) \leq \frac{1}{c_{1}} .
$$


Moreover, by (5.7) and the Paley-Zygmund inequality,

$$
\sup _{n \geq 1} \mathcal{W}\left(\mu_{n, \theta}(\overline{S(O, 1)})<\frac{1}{c_{1}}\right) \leq 1-\frac{\left(1-\frac{1}{c_{1}}\right)^{2}}{A_{1}} .
$$

Thus, by choosing $c_{1}$ and $c_{2}$ sufficiently large, we can make

$$
\mathcal{W}\left(\Lambda_{n}^{\alpha}\right)>\frac{\left(1-\frac{1}{c_{1}}\right)^{2}}{A_{1}}-\frac{1}{c_{1}}-\frac{A_{2}}{c_{2}}>\frac{1}{2 A_{1}}
$$

for every $n \geq 1$, and hence $\mathcal{W}\left(\Lambda^{\alpha}\right) \geq \frac{1}{2 A_{1}}$.

On the other hand, if $\theta \in \Lambda^{\alpha}$, then there exists a subsequence $\left\{n_{k}: k \geq 0\right\}$ such that

$$
\frac{1}{c_{1}} \leq \mu_{n_{k}, \theta}(\overline{S(O, 1)}) \leq c_{1}, I \quad \alpha\left(\mu_{n_{k}, \theta}\right) \leq c_{2} \quad \text { for all } k \geq 0
$$

Because $I_{\alpha}$, as a mapping from the space of finite measures on $\overline{S(O, 1)}$ to $[0, \infty]$ is lower semi-continuous with respect to the weak topology,

$$
\mathcal{M}:=\left\{\mu \text { Borel measure on } \overline{S(O, 1)}: \frac{1}{c_{1}} \leq \mu(\overline{S(O, 1)}) \leq c_{1}, I_{\alpha}(\mu) \leq c_{2}\right\}
$$

is compact, and hence there exists a Borel measure $\mu_{\theta}$ on $\overline{S(O, 1)}$ such that $\mu_{n_{k}, \theta}$ weakly converges to $\mu_{\theta}$ along a subsequence of $\left\{n_{k}: k \geq 0\right\}$. Without loss of generality, we can assume that the weak convergence happens along $\left\{n_{k}: k \geq 0\right\}$ itself. Thus,

$$
\frac{1}{c_{1}} \leq \mu_{\theta}(\overline{S(O, 1)}) \leq c_{1}, \quad I_{\alpha}\left(\mu_{\theta}\right) \leq c_{2} .
$$

Moreover, it is clear that for every $k \geq 0, \operatorname{supp}\left(\mu_{n_{k}, \theta}\right) \subseteq \overline{\bigcup_{x \in \Xi_{n_{k}, \theta}} S\left(x, r_{n_{k}}\right)}$. Combining this fact with the weak convergence relation, one can easily verify that

$$
\mu_{\theta}\left(\Sigma_{\theta}^{\gamma}\right) \geq \limsup _{k \rightarrow \infty} \mu_{n_{k}, \theta}\left(\overline{\bigcup_{x \in \Xi_{n_{k}, \theta}} S\left(x, r_{n_{k}}\right)}\right) \geq \frac{1}{c_{1}} .
$$

Therefore, if $\mathcal{C}^{\alpha}\left(\Sigma_{\theta}^{\gamma}\right)$ is the $\alpha$-capacity of the set $\Sigma_{\theta}^{\gamma}$, i.e.,

$$
\mathcal{C}^{\alpha}\left(\Sigma_{\theta}^{\gamma}\right):=\sup \left\{\left(\iint_{\Sigma_{\theta}^{\gamma} \times \Sigma_{\theta}^{\gamma}} \frac{\mu \times \mu(d y d w)}{|y-w|^{\alpha}}\right)^{-1}: \mu \text { is a probability measure on } \Sigma_{\theta}^{\gamma}\right\},
$$

then $\mathcal{C}^{\alpha}\left(\Sigma_{\theta}^{\gamma}\right)>0$. Hence, by Frostman's lemma, $\operatorname{dim}_{\mathcal{H}}\left(\Sigma_{\theta}^{\gamma}\right) \geq \alpha$ which implies that $\operatorname{dim}_{\mathcal{H}}\left(S T_{\theta}^{\gamma}\right) \geq \alpha$. Thus, we have established that

$$
\mathcal{W}\left(\operatorname{dim}_{\mathcal{H}}\left(S T_{\theta}^{\gamma}\right) \geq \alpha\right) \geq \mathcal{W}\left(\Lambda^{\alpha}\right) \geq \frac{1}{2 A_{1}} .
$$

In other words, $\operatorname{dim}_{\mathcal{H}}\left(S T_{\theta}^{\gamma}\right) \geq \alpha$ holds at least with a positive probability.

Finally, we recall from (2.1) that for $\mathcal{W}$-a.e. $\theta \in \Theta$,

$$
\theta=\sum_{n \geq 1} \mathcal{I}\left(h_{n}\right)(\theta) \cdot h_{n},
$$


where $\left\{h_{n}: n \geq 1\right\}$ is an orthonormal basis of the Cameron-Martin space $H$ and $\left\{\mathcal{I}\left(h_{n}\right): n \geq 1\right\}$ under $\mathcal{W}$ forms a sequence of i.i.d. standard Gaussian random variables. By a simple application of the Hewitt-Savage zero-one law, we have that

$$
\mathcal{W}\left(\operatorname{dim}_{\mathcal{H}}\left(S T_{\theta}^{\gamma}\right) \geq \alpha\right)=1 .
$$

Since $\alpha$ is arbitrary in $(0, v(1-\gamma))$, we get the desired lower bound, that is,

$$
\operatorname{dim}_{\mathcal{H}}\left(S T_{\theta}^{\gamma}\right) \geq v(1-\gamma) \text { a.s. }
$$

This completes the proof of Theorem 16. Since $S T_{\theta}^{\gamma}$ is a subset of $T_{\theta}^{\gamma}$, we have also established (4.3) and hence Theorem 9.

\section{Appendix}

In this appendix, we include the proofs of two technical results Lemmas 20 and 21 in Section 5.

Recall that, for Lemma 20, we want to show that, if for some $i \geq 0$, either

$$
r_{i}-r_{i+1}<\left|x_{j}^{(n)}-x_{k}^{(n)}\right| \leq r_{i}-r_{i+2},
$$

or

$$
r_{i}+r_{i+2} \leq\left|x_{j}^{(n)}-x_{k}^{(n)}\right|<r_{i}+r_{i+1},
$$

then

$$
\frac{\mathcal{W}\left(P_{x_{j}^{(n)}, i+1} \cap P_{x_{j}^{(n)}, i+2} \cap P_{x_{k}^{(n)}, i+1} \cap P_{x_{k}^{(n)}, i+2}\right)}{\mathcal{W}\left(P_{x_{j}^{(n)}, i+1}\right) \mathcal{W}\left(P_{x_{j}^{(n)}, i+2}\right) \mathcal{W}\left(P_{x_{k}^{(n)}, i+1}\right) \mathcal{W}\left(P_{x_{k}^{(n)}, i+2}\right)} \leq e^{C_{\nu} \sqrt{-\ln r_{i+1}}}
$$

for some constant $C_{v}>0$ that does not depend on $i$.

Proof. We will prove this result by multiple stages of conditioning. To further simplify the notation, throughout the proof, we write

$$
X_{i+1}:=\Delta \bar{\theta}_{i+1}\left(x_{j}^{(n)}\right), \quad X_{i+2}:=\Delta \bar{\theta}_{i+2}\left(x_{j}^{(n)}\right)
$$

and

$$
Y_{i+1}:=\Delta \bar{\theta}_{i+1}\left(x_{k}^{(n)}\right), \quad Y_{i+2}:=\Delta \bar{\theta}_{i+2}\left(x_{k}^{(n)}\right) .
$$

Clearly, $Y_{i+2}$ is independent of $Y_{i+1}$ and $X_{i+2}$. Furthermore, $\operatorname{Cov}\left(X_{i+1}, Y_{i+2}\right)$ is given by, when (3a) applies,

$$
\operatorname{DCov}(i+1, i+2)=-C_{\mathrm{incl}}\left(r_{i},\left|x_{j}^{(n)}-x_{k}^{(n)}\right|\right)+\operatorname{Cov}\left(r_{i}, x_{j}^{(n)} ; r_{i+1}, x_{k}^{(n)}\right)
$$

when (3b) applies,

$$
\operatorname{DCov}(i+1, i+2)=-C_{\mathrm{disj}}\left(\left|x_{j}^{(n)}-x_{k}^{(n)}\right|\right)+\operatorname{Cov}\left(r_{i}, x_{j}^{(n)} ; r_{i+1}, x_{k}^{(n)}\right) .
$$

In either case, $\operatorname{Cov}\left(X_{i+1}, Y_{i+2}\right)$ doesn't depend on $r_{i+2}$, and by the asymptotics of the functions that are involved and the Cauchy-Schwarz inequality,

$$
\operatorname{Cov}\left(X_{i+1}, Y_{i+2}\right)=\mathcal{O}\left(\sqrt{G\left(r_{i+1}\right) G\left(r_{i}\right)}\right) .
$$


Similarly, $\operatorname{Cov}\left(X_{i+1}, Y_{i+1}\right)$ is given by, when either (3a) or (3b) applies,

$$
\begin{aligned}
\operatorname{DCov}(i+1, i+1)= & C_{\mathrm{disj}}\left(\left|x_{j}^{(n)}-x_{k}^{(n)}\right|\right)-2 \operatorname{Cov}\left(r_{i}, x_{j}^{(n)} ; r_{i+1}, x_{k}^{(n)}\right) \\
& +\operatorname{Cov}\left(r_{i}, x_{j}^{(n)} ; r_{i}, x_{k}^{(n)}\right),
\end{aligned}
$$

which implies that

$$
\operatorname{Cov}\left(X_{i+1}, Y_{i+1}\right)=\mathcal{O}\left(\sqrt{G\left(r_{i+1}\right) G\left(r_{i}\right)}\right) .
$$

We first condition on $Y_{i+2}$. The joint conditional distribution of $\left\{X_{i+1}, X_{i+2}, Y_{i+1}\right\}$, given $Y_{i+2}=y$, is the same as the Gaussian family $\left\{X_{i+1}^{\prime}, X_{i+2}^{\prime}, Y_{i+1}^{\prime}\right\}$ where $X_{i+2}^{\prime}$ and $Y_{i+1}^{\prime}$ have the same distribution as $X_{i+2}$ and $Y_{i+1}$ respectively, and $X_{i+1}^{\prime}$ has the Gaussian distribution $N\left(m, \sigma^{2}\right)$ with

$$
m:=\frac{\operatorname{Cov}\left(X_{i+1}, Y_{i+2}\right)}{\Delta G_{i+2}} y \quad \text { and } \quad \sigma^{2}:=\Delta G_{i+1}-\frac{\operatorname{Cov}^{2}\left(X_{i+1}, Y_{i+2}\right)}{\Delta G_{i+2}} .
$$

In particular, if $\left|y-\sqrt{2 v \gamma} \Delta D_{i+2}\right| \leq \sqrt{\Delta G_{i+2}}$, then, by (5.1) and (A.1), $m=o(1)$ and $\sigma^{2}=\Delta G_{i+1}+o(1)$, and these estimates ${ }^{12}$ can be made uniform in $y$. Moreover, the covariance of the family is given by $\operatorname{Cov}\left(X_{i+1}^{\prime}, X_{i+2}^{\prime}\right)=0$, $\operatorname{Cov}\left(X_{i+2}^{\prime}, Y_{i+1}^{\prime}\right)=\operatorname{Cov}\left(X_{i+2}, Y_{i+1}\right)$ and $\operatorname{Cov}\left(X_{i+1}^{\prime}, Y_{i+1}^{\prime}\right)=\operatorname{Cov}\left(X_{i+1}, Y_{i+1}\right)$. We write the following conditional distribution as

$$
\mathcal{W}\left(P_{x_{j}^{(n)}, i+1} \cap P_{x_{j}^{(n)}, i+2} \cap P_{x_{k}^{(n)}, i+1} \mid Y_{i+2}=y\right)=\left.\mathcal{W}\right|_{Y_{i+2}=y}\left(P_{X_{i+1}^{\prime}} \cap P_{X_{i+2}^{\prime}} \cap P_{Y_{i+1}^{\prime}}\right),
$$

where $\left.\mathcal{W}\right|_{Y_{i+2}=y}$ is the conditional distribution under $\mathcal{W}$ given $Y_{i+2}=y$, and $P_{X_{i+1}^{\prime}}, P_{X_{i+2}^{\prime}}$ and $P_{Y_{i+1}^{\prime}}$ are the corresponding events concerning $X_{i+1}^{\prime}, X_{i+2}^{\prime}$ and $Y_{i+1}^{\prime}$, e.g.,

$$
P_{X_{i+1}^{\prime}}=\left\{\left|X_{i+1}^{\prime}-\sqrt{2 v \gamma} \Delta D_{i+1}\right| \leq \sqrt{\Delta G_{i+1}}\right\} .
$$

Next, we condition on $X_{i+2}^{\prime}=x$ where $\left|x-\sqrt{2 \nu \gamma} \Delta D_{i+2}\right| \leq \sqrt{\Delta G_{i+2}}$. Then the conditional distribution of $\left\{X_{i+1}^{\prime}, Y_{i+1}^{\prime}\right\}$ is the same as that of $\left\{X_{i+1}^{\prime \prime}, Y_{i+1}^{\prime \prime}\right\}$ where $X_{i+1}^{\prime \prime}$ has the same distribution as $X_{i+1}^{\prime}$, and $Y_{i+1}^{\prime \prime}$ has the Gaussian distribution $N\left(\lambda, \varsigma^{2}\right)$ where

$$
\lambda=\frac{\operatorname{Cov}\left(X_{i+2}, Y_{i+1}\right)}{\Delta G_{i+2}} x \quad \text { and } \quad \varsigma^{2}=\Delta G_{i+1}-\frac{\operatorname{Cov}^{2}\left(X_{i+2}, Y_{i+1}\right)}{\Delta G_{i+2}} .
$$

Since $\operatorname{Cov}\left(X_{i+2}, Y_{i+1}\right)=\operatorname{Cov}\left(X_{i+1}, Y_{i+2}\right)$, the estimates we obtained for $m$ and $\sigma^{2}$ also applies to $\lambda$ and $\varsigma^{2}$ respectively, and those estimates are uniform in $x$ and $y$. In addition, $\operatorname{Cov}\left(X_{i+1}^{\prime \prime}, Y_{i+1}^{\prime \prime}\right)=\operatorname{Cov}\left(X_{i+1}, Y_{i+1}\right)$. Again, we write the following conditional distribution as

$$
\left.\mathcal{W}\right|_{Y_{i+2}=y}\left(P_{X_{i+1}^{\prime}} \cap P_{Y_{i+1}^{\prime}} \mid X_{i+2}^{\prime}=x\right)=\left.\mathcal{W}\right|_{X_{i+2}^{\prime}=x}\left(P_{X_{i+1}^{\prime \prime}} \cap P_{Y_{i+1}^{\prime \prime}}\right),
$$

where $\left.\mathcal{W}\right|_{X_{i+2}^{\prime}=x}$ is the conditional distribution under $\left.\mathcal{W}\right|_{Y_{i+2}=y}$ conditioning on $X_{i+2}^{\prime}=x$, and $P_{X_{i+1}^{\prime \prime}}$ and $P_{Y_{i+1}^{\prime \prime}}$ are the corresponding events concerning $X_{i+1}^{\prime \prime}$ and $Y_{i+1}^{\prime \prime}$.

To compute $\left.\mathcal{W}\right|_{X_{i+2}^{\prime}=x}\left(P_{X_{i+1}^{\prime \prime}} \cap P_{Y_{i+1}^{\prime \prime}}\right)$, we use conditioning again. Given

$$
Y_{i+1}^{\prime \prime}=w \in\left[\sqrt{2 \nu \gamma} \Delta D_{i+1}-\sqrt{\Delta G_{i+1}}, \sqrt{2 v \gamma} \Delta D_{i+1}+\sqrt{\Delta G_{i+1}}\right],
$$

the conditional distribution of $X_{i+1}^{\prime \prime}$ is the Gaussian distribution with the mean

$$
m+\frac{\operatorname{Cov}\left(X_{i+1}, Y_{i+1}\right)}{\varsigma^{2}}(w-\lambda)=\mathcal{O}\left(\sqrt{G\left(r_{i}\right)\left(-\ln r_{i+1}\right)}\right)
$$

\footnotetext{
${ }^{12}$ Here, as well as in later occasions, when concerning $o(1)$, the "estimate" refers to the rate of the $o(1)$ term converging to zero.
} 
and the variance

$$
\sigma^{2}-\frac{\operatorname{Cov}^{2}\left(X_{i+1}, Y_{i+1}\right)}{\varsigma^{2}}=\Delta G_{i+1}(1+o(1)) .
$$

These estimates ${ }^{13}$ follow from (A.2) and earlier estimates on $m, \lambda, \sigma^{2}$ and $\varsigma^{2}$, and they can be made uniform in $w, x$ and $y$. Therefore, one can easily verify that

$$
\left.\mathcal{W}\right|_{X_{i+2}^{\prime}=x}\left(P_{X_{i+1}^{\prime \prime}} \mid Y_{i+1}^{\prime \prime}=w\right) \leq \exp \left[\nu \gamma \ln r_{i+1}+\mathcal{O}\left(\sqrt{-\ln r_{i+1}}\right)\right]:=p_{1},
$$

and $p_{1}$ is independent of $w, x$ and $y$. This further leads to

$$
\begin{aligned}
\left.\mathcal{W}\right|_{X_{i+2}^{\prime}=x}\left(P_{X_{i+1}^{\prime \prime}} \cap P_{Y_{i+1}^{\prime \prime}}\right) & \leq p_{1} \exp \left[\nu \gamma \ln r_{i+1}+\mathcal{O}\left(\sqrt{-\ln r_{i+1}}\right)\right] \\
& =\exp \left[2 \nu \gamma \ln r_{i+1}+\mathcal{O}\left(\sqrt{-\ln r_{i+1}}\right)\right]:=p_{2},
\end{aligned}
$$

and $p_{2}$ is independent of $x$ and $y$.

Finally, since $X_{i+2}^{\prime}$ has the same distribution as $X_{i+2}$, by backtracking the condition, we have that

$$
\left.\mathcal{W}\right|_{Y_{i+2}=y}\left(P_{X_{i+1}^{\prime}} \cap P_{Y_{i+1}^{\prime}} \cap P_{X_{i+2}^{\prime}}\right) \leq p_{2} \mathcal{W}\left(P_{x_{j}^{(n)}, i+2}\right),
$$

and hence

$$
\mathcal{W}\left(P_{x_{j}^{(n)}, i+1} \cap P_{x_{j}^{(n)}, i+2} \cap P_{x_{k}^{(n)}, i+1} \cap P_{x_{k}^{(n)}, i+2}\right) \leq p_{2} \mathcal{W}\left(P_{x_{j}^{(n)}, i+2}\right) \mathcal{W}\left(P_{x_{k}^{(n)}, i+2}\right) .
$$

The desired estimate follows immediately from (5.3).

Next, for Lemma 21, we want to show that, if for some $i \geq 0$, either

$$
r_{i+1}^{1-\epsilon}<\left|x_{j}^{(n)}-x_{k}^{(n)}\right| \leq r_{i}-r_{i+1},
$$

or

$$
r_{i}+r_{i+1} \leq\left|x_{j}^{(n)}-x_{k}^{(n)}\right|<2 r_{i},
$$

then

$$
\frac{\mathcal{W}\left(P_{x_{j}^{(n)}, i} \cap P_{x_{j}^{(n)}, i+1} \cap P_{x_{k}^{(n)}, i+1}\right)}{\mathcal{W}\left(P_{x_{j}^{(n)}, i}\right) \mathcal{W}\left(P_{x_{j}^{(n)}, i+1}\right) \mathcal{W}\left(P_{x_{k}^{(n)}, i+1}\right)} \leq \exp \left(C_{\mathcal{v}, \epsilon} \sqrt{-\ln r_{i}}\right)
$$

for some constant $C_{v, \epsilon}>0$ that does not depend on $i$.

Proof. The proof of Lemma 21 is similar to the proof of Lemma 20 given above. Namely, we will also prove the desired estimate by multiples stages of conditioning. For simpler notation, we write

$$
X_{i}:=\Delta \bar{\theta}_{i}\left(x_{j}^{(n)}\right), \quad X_{i+1}:=\Delta \bar{\theta}_{i+1}\left(x_{j}^{(n)}\right) \quad \text { and } \quad Y_{i+1}:=\Delta \bar{\theta}_{i+1}\left(x_{k}^{(n)}\right) .
$$

When (4a) or (4b) applies, by (3.7) and (3.8),

$$
\operatorname{Cov}\left(X_{i}, Y_{i+1}\right)=\mathcal{O}\left(G\left(r_{i}\right)\right)
$$

\footnotetext{
${ }^{13}$ Here, as well as in later occasions, when concerning " $\mathcal{O}$ ", the "estimate" refers to the constants in the upper and lower bound.
} 
and

$$
\operatorname{Cov}\left(Y_{i+1}, X_{i+1}\right)=\mathcal{O}\left(G^{1-\epsilon}\left(r_{i+1}\right)\right) .
$$

We first condition on $Y_{i+1}=y$ where $\left|y-\sqrt{2 v \gamma} \Delta D_{i+1}\right| \leq \sqrt{\Delta G_{i+1}}$. Then the joint conditional distribution of $\left\{X_{i}, X_{i+1}\right\}$ given $Y_{i+1}=y$ is the same as that of $\left\{X_{i}^{\prime}, X_{i+1}^{\prime}\right\}$ where $X_{i}^{\prime}$ and $X_{i+1}^{\prime}$ have distributions $N\left(m_{1}, \sigma_{1}^{2}\right)$ and $N\left(m_{2}, \sigma_{2}^{2}\right)$ respectively, where $m_{1}=o(1), \sigma_{1}^{2}=\Delta G_{i}+o(1)$, and

$$
m_{2}=\mathcal{O}\left(\Delta D_{i+1} \cdot G^{-\epsilon}\left(r_{i+1}\right)\right) \quad \text { and } \quad \sigma_{2}^{2}=\Delta G_{i+1}\left[1+\mathcal{O}\left(G^{-2 \epsilon}\left(r_{i+1}\right)\right)\right],
$$

and moreover,

$$
\operatorname{Cov}\left(X_{i}^{\prime}, X_{i+1}^{\prime}\right)=\mathcal{O}\left(G\left(r_{i}\right) / G^{\epsilon}\left(r_{i+1}\right)\right) .
$$

These estimates on $m_{1}, \sigma_{1}^{2}, m_{2}, \sigma_{2}^{2}$ and $\operatorname{Cov}\left(X_{i}^{\prime}, X_{i+1}^{\prime}\right)$ follow from (A.3) and (A.4) and can be made uniform in $y$. Next, we condition on $X_{i}^{\prime}=x$ where $\left|x-\sqrt{2 v \gamma} \Delta D_{i}\right| \leq \sqrt{\Delta G_{i}}$. Then the conditional distribution of $X_{i+1}^{\prime}$ is the Gaussian distribution $N\left(m_{3}, \sigma_{3}^{2}\right)$ and, by (A.6), $m_{3}$ and $\sigma_{3}^{2}$ follow the same estimates as $m_{2}$ and $\sigma_{2}^{2}$ respectively, i.e., the estimates in (A.5), and these estimates are uniform in $x$ and $y$.

To proceed from here, we need to carry out a step that is different from the proof of Lemma 20. Specifically, we need to compare $\mathcal{W}\left(P_{x_{j}^{(n)}, i+1}\right)$ and

$$
N\left(m_{3}, \sigma_{3}^{2}\right)\left(\left[\sqrt{2 v \gamma} \Delta D_{i+1}-\sqrt{\Delta G_{i+1}}, \sqrt{2 v \gamma} \Delta D_{i+1}+\sqrt{\Delta G_{i+1}}\right]\right) .
$$

To this end, we write the later as

$$
\begin{aligned}
& \frac{1}{\sqrt{2 \pi}} \int_{\sqrt{2 v \gamma} \Delta D_{i+1}-\sqrt{\Delta G_{i+1}}}^{\sqrt{2 v \gamma} \Delta D_{i+1}+\sqrt{\Delta G_{i+1}}} \frac{\exp \left[-\left(w-m_{3}\right)^{2} /\left(2 \sigma_{3}^{2}\right)\right]}{\sigma_{3}} d w \\
& \quad=\frac{1}{\sqrt{2 \pi}} \int_{\sqrt{2 v \gamma} \Delta D_{i+1}-\sqrt{\Delta G_{i+1}}}^{\sqrt{2 \nu \gamma} \Delta D_{i+1}+\sqrt{\Delta G_{i+1}}} \frac{\exp \left[-w^{2} /\left(2 \Delta G_{i+1}\right)\right]}{\sqrt{\Delta G_{i+1}}} \cdot E(w) d w
\end{aligned}
$$

where

$$
E(w):=\frac{\sqrt{\Delta G_{i+1}}}{\sigma_{3}} \exp \left[-\frac{\left(w-m_{3}\right)^{2}}{2 \sigma_{3}^{2}}+\frac{w^{2}}{2 \Delta G_{i+1}}\right] .
$$

Notice that by the estimates in (A.5) which apply to $m_{3}$ and $\sigma_{3}^{2}$, there exists a constant $C_{v, \epsilon}>0$ such that

$$
\sup _{\left\{w:\left|w-\sqrt{2 v \gamma} \Delta D_{i+1}\right| \leq \sqrt{\Delta G_{i+1}}\right\}}|E(w)| \leq C_{\nu, \epsilon} .
$$

It follows from this observation that, conditioning on $X_{i}^{\prime}=x$, the conditional probability of $X_{i+1}^{\prime}$ being in the desired interval, i.e., $\left|X_{i+1}^{\prime}-\sqrt{2 v \gamma} \Delta D_{i+1}\right| \leq \sqrt{\Delta G_{i+1}}$, is bounded by $C_{v, \epsilon} \mathcal{W}\left(P_{x_{j}^{(n)}, i+1}\right)$. From this point, we backtrack the conditioning in the same way as we did in the proof of Lemma 20 and arrive at

$$
\mathcal{W}\left(P_{x_{j}^{(n)}, i} \cap P_{x_{j}^{(n)}, i+1} \cap P_{x_{k}^{(n)}, i+1}\right) \leq e^{\nu \gamma \ln r_{i}+\mathcal{O}\left(\sqrt{-\ln r_{i}}\right)} \cdot \mathcal{W}\left(P_{x_{j}^{(n)}, i+1}\right) \mathcal{W}\left(P_{x_{k}^{(n)}, i+1}\right) .
$$

By (5.3), the desired estimate follows immediately.

\section{Acknowledgement}

The author would like to thank Daniel Stroock for helpful discussions. 


\section{References}

[1] R. Adler and J. Taylor. Random Fields and Geometry. Springer, New York, 2007. MR2319516

[2] J. Barral and B. Mandelbrot. Non-degeneracy, moments, dimension, and multifractal analysis for random multiplicative measures (random multiplicative multifractal measures, part ii). Proc. Symp. Pure Math., AMS 72 (2004) 17-52. MR2112120

[3] J. Barral, R. Rhodes, X. Jin and V. Vargas. Gaussian multiplicative chaos and kpz duality. Commun. Math. Phys. 323 (2013) $451-485$.

[4] S. Chatterjee, A. Dembo and J. Ding. On level sets of Gaussian fields 1-6, 2013. Available at arXiv:1310.5175v1.

[5] L. Chen and D. Jakobson. Gaussian free fields and kpz relation in $\mathbb{R}^{4}$. Ann. Henri Poincaré 15 (7) (2014) 1245-1283. MR3225731

[6] L. Chen and D. Stroock. Additive functions and Gaussian measures. In Prokhorov and Contemporary Probability Theory. Springer Proceedings in Mathematics and Statistics 33, 2013. MR3070474

[7] A. Cipriani and R. S. Hazra. Thick points for Gaussian free fields with different cut-offs 1-32, 2014. Available at arXiv:1407.5840v1. MR3606735

[8] A. Cipriani and R. S. Hazra. Thick points for a Gaussian free fields in 4 dimensions. Stochastic Process. Appl. 125 (6) (2015) $2383-2404$. MR3322868

[9] A. Dembo, Y. Peres, J. Rosen and O. Zeitouni. Thick points for spatial Brownian motion: Multifractal analysis of occupation measure. Ann. Probab. 28 (1) (2000) 1-35. MR1755996

[10] J. Ding, R. Roy and O. Zeitouni. Convergence of the centered maximum of log-correlated Gaussian fields. Ann. Probab. To appear. Available at arXiv:1503.04588v1.

[11] J. Ding and O. Zeitouni. Extreme values for two-dimensional discrete Gaussian free field. Ann. Probab. 42 (4) (2014) $1480-1515$. MR3262484

[12] A. Drewitz and P. Rodriguez. High-dimensional asymptotics for percolation of Gaussian free field level sets. Electron. J. Probab. 20 (47) (2015) 1-39. MR3339867

[13] R. Dudley. Sample functions of the Gaussian process. Ann. Probab. 1 (1973) 66-103. MR0346884

[14] B. Duplantier, R. Rhodes, S. Sheffield and V. Vargas. Renormalization of critical Gaussian multiplicative chaos and kpz relation. Comm. Math. Phys. 330 (1) (2014) 283-330. MR3215583

[15] B. Duplantier and S. Sheffield. Liouville quantum gravity and kpz. Invert. Math. 185 (2) (2011) 333-393. MR2819163

[16] L. Gross. Abstract Wiener spaces. In Proc. 5th Berkeley Symp. Math. Stat. and Probab. 2, 1965 31-42. MR0212152

[17] X. Hu, J. Miller and Y. Peres. Thick points of the Gaussian free field. Ann. Probab. 38 (2) (2010) 896-926. MR2642894

[18] J.-P. Kahane. Random Series of Functions, 2nd edition. Cambridge Studies in Advanced Mathematics 5. Cambridge Univ. Press, Cambridge, 1985. MR0833073

[19] T. Madaule. Maximum of a log-correlated Gaussian field. Ann. Inst. Henri Poincaré 51 (4) (2015) 1369-1431. MR3414451

[20] R. Rhodes and V. Vargas. Multidimensional multifractal random measures. Electron. J. Probab. 15 (9) (2010) 241-258. MR2609587

[21] R. Rhodes and V. Vargas. Kpz formula for log-infinitely divisible multifractal random measures. ESAIM Probab. Stat. 15 (2011) $358-371$. MR2870520

[22] R. Rhodes and V. Vargas. Gaussian multiplicative chaos and applications: A review, 2013. Available at arXiv:1305.6221v1. MR3274356

[23] S. Sheffield. Gaussian free fields for mathematicians. Probab. Theory Related Fields 139 (3-4) (2007) 521-541. MR2322706

[24] D. Stroock. Abstract Wiener space, revisited. Commun. Stoch. Anal. 2 (1) (2008) 145-151. MR2446996

[25] D. Stroock. Probability, an Analytic View, 2nd edition. Cambridge Univ. Press, Cambridge, 2011. MR1267569

[26] M. Talagrand. Majorizing measures: The generic chaining. Ann. Probab. 24 (1996) 1049-1103. MR1411488

[27] G. N. Watson. A Treatise on the Theory of Bessel Functions, 2nd edition. Cambridge Univ. Press, Cambridge, 1995. MR1349110 